\title{
PD-1 blockade improves Kupffer cell bacterial clearance in acute liver injury
}

\author{
Evangelos Triantafyllou, ${ }^{1,2}$ Cathrin L.C. Gudd, ${ }^{1,2}$ Marie-Anne Mawhin, ${ }^{2}$ Hannah C. Husbyn, ${ }^{1}$ Francesca M. Trovato, ${ }^{3}$ \\ Matthew K. Siggins, ${ }^{4}$ Thomas O'Connor, ${ }^{1}$ Hiromi Kudo, ${ }^{5}$ Sujit K. Mukherjee, Julia A. Wendon, ${ }^{3}$ Christine Bernsmeier, \\ Robert D. Goldin, ${ }^{5}$ Marina Botto, ${ }^{2}$ Wafa Khamri, ${ }^{1}$ Mark J.W. McPhail, ${ }^{3}$ Lucia A. Possamai, ${ }^{1}$ Kevin J. Woollard, ${ }^{2}$ \\ Charalambos G. Antoniades, ${ }^{1}$ and Mark R. Thursz ${ }^{1}$
}

DDepartment of Metabolism, Digestion and Reproduction, Section of Hepatology and Gastroenterology, and 2Department of Immunology and Inflammation, Centre for Inflammatory Disease, Imperial College London, London, United Kingdom. ${ }^{3}$ Division of Transplantation Immunology and Mucosal Biology, King's College London, London, United Kingdom. ${ }^{4}$ Department of Infectious Disease, and ${ }^{5}$ Department of Metabolism, Digestion and Reproduction, Section of Pathology, Imperial College London, London, United Kingdom. ${ }^{6}$ Department of Biomedicine, University of Basel and University Centre for Castrointestinal and Liver Diseases, Basel, Switzerland.

Patients with acute liver failure (ALF) have systemic innate immune suppression and increased susceptibility to infections. Programmed cell death 1 (PD-1) expression by macrophages has been associated with immune suppression during sepsis and cancer. We therefore examined the role of the programmed cell death 1/programmed death ligand 1 (PD-1/PD-L1) pathway in regulating Kupffer cell (KC) inflammatory and antimicrobial responses in acetaminophen-induced (APAP-induced) acute liver injury. Using intravital imaging and flow cytometry, we found impaired KC bacterial clearance and systemic bacterial dissemination in mice with liver injury. We detected increased PD-1 and PD-L1 expression in KCs and lymphocyte subsets, respectively, during injury resolution. Gene expression profiling of $\mathrm{PD}-1^{+} \mathrm{KC}$ s revealed an immune-suppressive profile and reduced pathogen responses. Compared with WT mice, PD-1-deficient mice and anti-PD-1-treated mice with liver injury showed improved KC bacterial clearance, a reduced tissue bacterial load, and protection from sepsis. Blood samples from patients with ALF revealed enhanced PD-1 and PD-L1 expression by monocytes and lymphocytes, respectively, and that soluble PD-L1 plasma levels could predict outcomes and sepsis. PD-1 in vitro blockade restored monocyte functionality. Our study describes a role for the PD-1/PD-L1 axis in suppressing KC and monocyte antimicrobial responses after liver injury and identifies anti-PD-1 immunotherapy as a strategy to reduce infection susceptibility in ALF.

\section{Introduction}

Acute liver failure (ALF) is a rare clinical syndrome in which coagulopathy, jaundice, and hepatic encephalopathy arise in the context of acute hepatic injury without preexisting chronic liver disease (CLD) $(1,2)$. Acetaminophen (APAP) overdose is the most common cause of ALF in the United Kingdom and the United States $(1,2)$. Immune dysfunction is central to the pathogenesis of ALF. Although the initiating event in ALF is overwhelming hepatocyte death, mortality occurs as a result of profound activation of hepatic and systemic inflammatory responses and the associated complications of multiorgan failure, immune paresis, and sepsis (3).

Bacterial infections are common complications of ALF, occurring late ( $>5$ days after hospital admission) in $35 \%-40 \%$ of patients and are considered a leading cause of mortality (3-5). This is a consequence of ALF-related immune paresis and the

Authorship note: CGA is deceased. CGA and MRT are co-senior authors.

Conflict of interest: The authors have declared that no conflict of interest exists. Copyright: (5) 2021, Triantafyllou et al. This is an open access article published under the terms of the Creative Commons Attribution 4.0 International License.

Submitted: May 13, 2020; Accepted: December 10, 2020; Published: February 15, 2021. Reference information: J Clin Invest. 2021;131(4):e140196.

https://doi.org/10.1172/JCl140196. invasive nature of critical care support that predisposes patients to nosocomial infections (3). Our group has previously demonstrated defects in both innate and adaptive systemic immunity in ALF (48). Monocytes of patients with ALF are hyporesponsive to microbial challenge and show reduced proinflammatory cytokine secretion, impaired Escherichia coli bacterial uptake, and an M2-like phenotype that favors the resolution of inflammation $(4-6,8)$.

Macrophages are central to maintaining liver homeostasis but are equally relevant in responses to injury or infection $(3,9)$. Liver-resident macrophages, or Kupffer cells (KCs), have an embryonic origin and a capacity for self-renewal $(3,9)$. KCs reside within liver sinusoids and function as the dominant intravascular phagocytes with a fundamental role in the clearance of blood-borne bacteria, a process that is critical for the prevention of disseminated infections (10-13). After APAP-induced liver injury in mice, a reduction in KCs occurs 24-48 hours after APAP, and full recovery takes place by self-renewal $(6,14-16)$. The total macrophage pool is expanded during resolution (APAP, $72 \mathrm{~h}$ ) via recruitment of bone marrow monocytes that differentiate in situ into monocyte-derived macrophages (MoMFs) (14-17). Human and mouse studies indicate that KCs and MoMFs have both tissuedestructive and pro-restorative/tissue repair functions in APAP injury $(6,14-16)$. However, the role of KCs in mediating antimicrobial responses after liver injury remains unexplored. 
Programmed cell death 1 (PD-1) plays an essential role in balancing protective immunity and immunopathology, homeostasis, and tolerance. However, during chronic infections and cancer, it can limit protective immunity (18). PD-1 is a major regulator of $\mathrm{T}$ cell responses, expressed on activated $\mathrm{T}$ cells, $\mathrm{B}$ cells, and Tregs $(18,19)$. PD-1 interacts with its ligands, PD-L1 and PDL-2, and their engagement triggers downstream signaling. Immune checkpoint inhibition using $\mathrm{mAbs}$ against $\mathrm{PD}-1 / \mathrm{PD}-\mathrm{L} 1$ has revolutionized cancer treatment, with significant clinical success in some patients $(18,19)$. In an effort to increase the therapeutic reach of targeting this pathway, the role of PD- 1 has been examined in DCs, NK cells, and innate lymphoid cells (20).

Expression in myeloid cells of PD-1 and its potential for therapeutic targeting have also started to be appreciated. PD-1 can be induced in monocytes and macrophages through TLR signaling $(21,22)$. Furthermore, PD-1 expression by macrophages plays a pathologic role by suppressing innate inflammatory responses in sepsis and inhibiting phagocytosis in active tuberculosis (23, 24). In the context of cancer, PD-1 expression in macrophages inversely correlates with M1-like polarization and the phagocytic potency of tumor-associated macrophages (TAMs) against tumor $(25,26)$. PD-1 expression in myeloid cells was very recently shown to dampen antitumor immunity by regulating myeloid cell lineage fate commitment and function (27). However, the role of this pathway in regulating macrophage responses during acute liver injury remains unknown.

In this study, we first aimed to examine KC bacterial clearance in mice with APAP-induced acute liver injury. Second, we sought to investigate the role of the PD-1/PD-L1 pathway in regulating $\mathrm{KC}$ inflammatory and antimicrobial responses after injury. This was further assessed in blood and liver tissue samples from patients with ALF. We provide evidence that following injury, KCs display impaired bacterial clearance, a suppressive effect that is mediated via the PD-1/PD-L1 axis. Our work identifies PD-1targeted immunotherapy as a strategy to reduce susceptibility to infections in liver failure.

\section{Results}

Reduced KC bacterial clearance and increased bacterial dissemination in mice with acute liver injury. To examine the functional status of KCs in mediating bacterial clearance in the liver, we studied untreated (control) and APAP-treated ( $72 \mathrm{~h}$ : resolution phase) mice following systemic E. coli infection (Figure 1A). Using liver intravital imaging to visualize the dynamic interactions between KCs and circulating E. coli bacteria, we found that E. coli were arrested in the liver within 20 minutes of infection (Figure 1, B and $\mathrm{C}$ ), in line with the crucial role of KCs in clearing blood-borne infections (10-13). E. coli bacteria colocalized with F4/80-labeled KCs (Figure 1B), while 3D reconstitution with increased KC transparency confirmed that captured E. coli were inside KCs (Supplemental Videos 1 and 2; supplemental material available online with this article; https://doi.org/10.1172/JCI140196DS1).

Compared with controls, APAP-treated mice showed fewer captured $E$. coli in the liver (20 min after infection, Figure 1, B and $\mathrm{C}$ ). KC numbers were similar between study groups (Figure 1D and Supplemental Figure 1A), suggesting that the decreased E. coli capture after liver injury was due to a qualitative defect in KC phagocytosis rather than a quantitative deficiency in KCs. To test this, we performed flow cytometry and found reduced E. coli uptake in KCs isolated from infected APAP-treated mice compared with controls (Figure 1D). We further examined bacteria internalization by KCs using intravital imaging in mice challenged with pHrodo $E$. coli, a pH-sensitive assay indicating phagolysosome acidification, which confirmed a reduced KC bacterial uptake capacity in APAP-treated mice (Supplemental Figure 1, B and C). In addition, KCs from APAP-treated mice were characterized by a decrease in bacterial killing ability, as indicated by the greater number of viable E. coli recovered from KCs (Figure 1E and Supplemental Figure 1E).

We further quantified the number of bacteria in the blood and different organs (20 min after infection). Compared with controls, APAP-treated mice displayed significantly higher blood E. coli counts (Figure $1 \mathrm{~F}$ ) and increased bacterial burden in the spleen, lungs, and kidneys (Supplemental Figure 1, D and E). Together, these data suggest reduced $\mathrm{KC}$ bacterial clearance and systemic bacterial dissemination in mice with acute liver injury.

Increased PD-1 and PD-L1 expression in KCs after acute liver injury in mice. Given the implication of the PD-1/PD-L1 axis in macrophage suppression during sepsis and cancer $(23,26)$, we measured the levels of PD-1 and PD-L1 expression by macrophages during murine acute liver injury. We observed peak injury at 24 hours and resolution of inflammation within 72 hours of APAP treatment (refs. 6, 14 and Supplemental Figure 1F). Flow cytometric analysis of liver KCs and MoMFs (Figure 2A) showed a reduction of KCs at 48 hours with full recovery by 72 hours, as previously reported (14-16), whereas liver recruitment of MoMFs contributed to the expansion of total macrophages at 72 hours (Figure 2B and Supplemental Figure 2A). Both PD-1 and PD-L1 were expressed in KCs and MoMFs at baseline (Figure $2, \mathrm{C}-\mathrm{F}$ ), and the total number of $\mathrm{PD}-1^{+}$and $\mathrm{PD}-\mathrm{L}^{+}$liver macrophages increased after injury (Figure 2, C and E, and Supplemental Figure 2, B and C). Notably, PD-1 and PD-L1 expression was increased in KCs during the resolution phase, whereas only PD-L1 expression was upregulated in MoMFs (Figure 2, D and F). In line with these data, liver Pdl1 mRNA was upregulated at 72 hours (Supplemental Figure 1G).

Increased PD-L1 expression of lymphocyte subsets after acute liver injury in mice. PD-1 signaling inhibits T cell activation, while PD-L1 ${ }^{+}$ $\mathrm{T}$ cells can also induce tolerogenic macrophages via PD-1 ligation (28). To explore this in our context, we determined the PD-1 and PD-L1 expression levels in lymphocyte subsets during murine acute liver injury. Flow cytometric analysis revealed an expansion of liver $\mathrm{CD}^{+} \mathrm{T}$ cells, $\mathrm{CD} 8^{+} \mathrm{T}$ cells, and Tregs during the resolution phase (72 h) (Figure 3, A and B, and Supplemental Figure 2D). PD-1 expression in these cell subsets remained unaltered, whereas PD-L1 expression in Tregs was upregulated 72 hours after APAP (Figure 3D and Supplemental Figure 2F). In addition, flow cytometric analysis of NK and NKT cells revealed an expansion of NKTs during resolution (Figure 3, A and C, and Supplemental Figure 2E). NK cell PD-1 and PD-L1 expression remained unaltered (Figure 3E and Supplemental Figure 2G), whereas PD-L1 expression in NKT cells was upregulated at 72 hours (Figure 3E). In line with this, liver tissue mRNA levels of Pdl1 and the lymphocyte recruitment chemokines Cxcl1O and Cxcl16 were upregulated at 72 hours (Supplemental Figure 1G). 
A
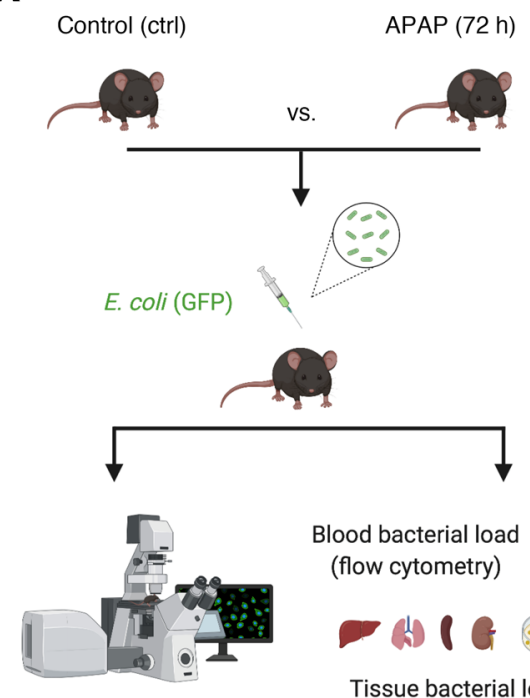

Liver intravital imaging (20 min after infection)
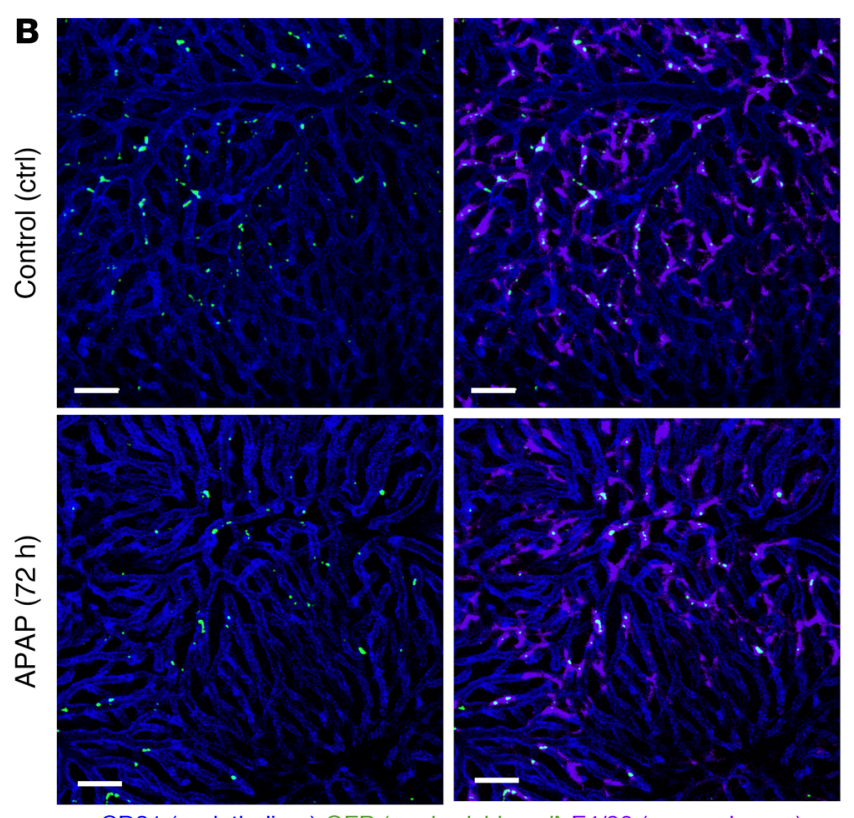

CD31 (endothelium) GFP (escherichia coli) F4/80 (macrophages)

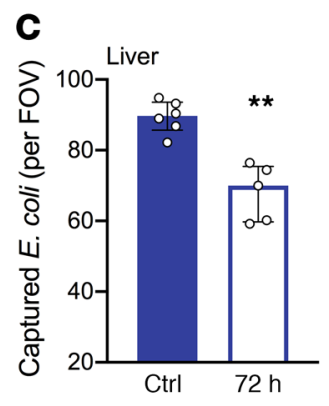

D
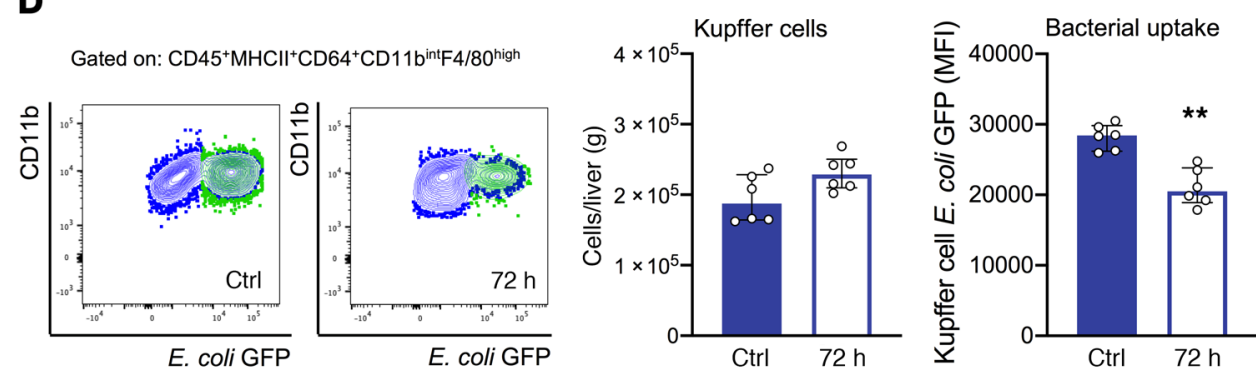

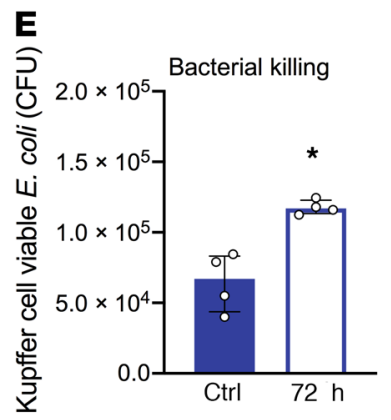

$\mathbf{F}$
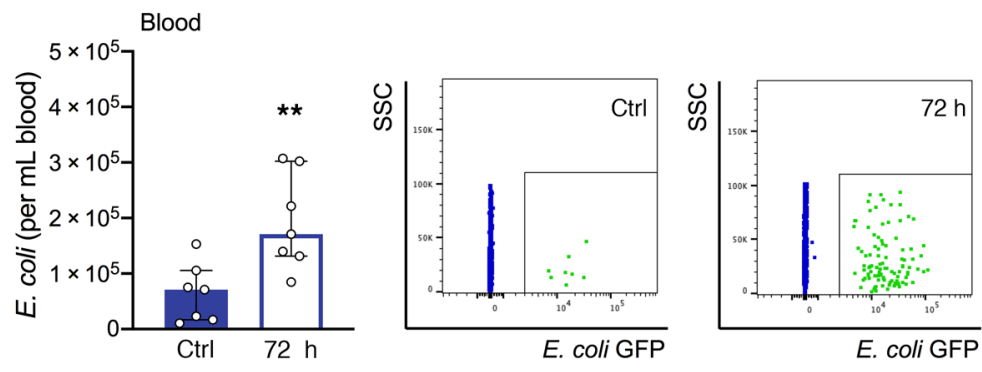

Figure 1. KC bacterial clearance is reduced in mice with acute liver injury. Baseline (control [ctrl]) and APAP-treated (72 h) mice were intravenously challenged with E. coli. (A) Schematic of experimental E. coli challenge in mice. (B) Representative intravital liver images (20 min after infection). Macrophages and endothelial cells were stained with fluorescently labeled anti-F4/80 (purple) and anti-CD31 (blue) antibodies, respectively; GFP+ E. coli (green). Scale bars: $50 \mu \mathrm{m}$. (C) Intravital imaging analysis shows the amount of macrophage-captured $E$. coli per FOV ( $n=5-6$ per group). (D) Representative flow plots of KC (blue) E. coli (green) capture measured by flow cytometry. Dot plots show KC numbers and $E$. coli uptake as measured by flow cytometry ( $n=$ 6 per group). (E) Sorted KCs from control and APAP-treated ( $72 \mathrm{~h}$ ) mice were in vitro challenged with $E$. coli (1:100 ratio, $60 \mathrm{~min})$. In vitro bacterial killing of KC-phagocytosed $E$. coli was evaluated by measuring viable $E$. coli CFU recovered from KC lysates ( $n=4$ per group). (F) Representative dot plots and number of free GFP+ . coli in blood measured by flow cytometry ( $n=7$ per group). SSC, side scatter. Results are from 3 (B-D, and F) and 2 (E) independent experiments. Each symbol represents an individual mouse. Data are presented as the median with the IQR. ${ }^{*} P<0.05$ and ${ }^{* *} P<0.01$, by Mann-Whitney $U$ test.

PD-1-expressing KCs exhibit an immune-suppressive profile in acute liver injury. To fully characterize the PD-1+ KCs during resolution of the injury, flow cytometry-sorted PD-1+ ${ }^{+}$and PD-1- KCs (72 $\mathrm{h}$ after APAP) were subjected to mRNA expression analysis (Figure 4A, Supplemental Figure 3, and Supplemental Data File 1). Compared with PD-1- KCs, PD-1 $1^{+}$KCs had increased scores for angiogenesis, metabolism, extracellular matrix (ECM) remodeling, and cytokine signaling (Figure 4B and Supplemental Figure 3B). In contrast, pathway scores for lymphocyte activation, antigen presentation, pathogen responses, and chemokine, IFN, TLR, and $\mathrm{Fc}$ receptor signaling were reduced in $\mathrm{PD}-1^{+} \mathrm{KCs}$ (Figure 4B and Supplemental Figure 3B). 

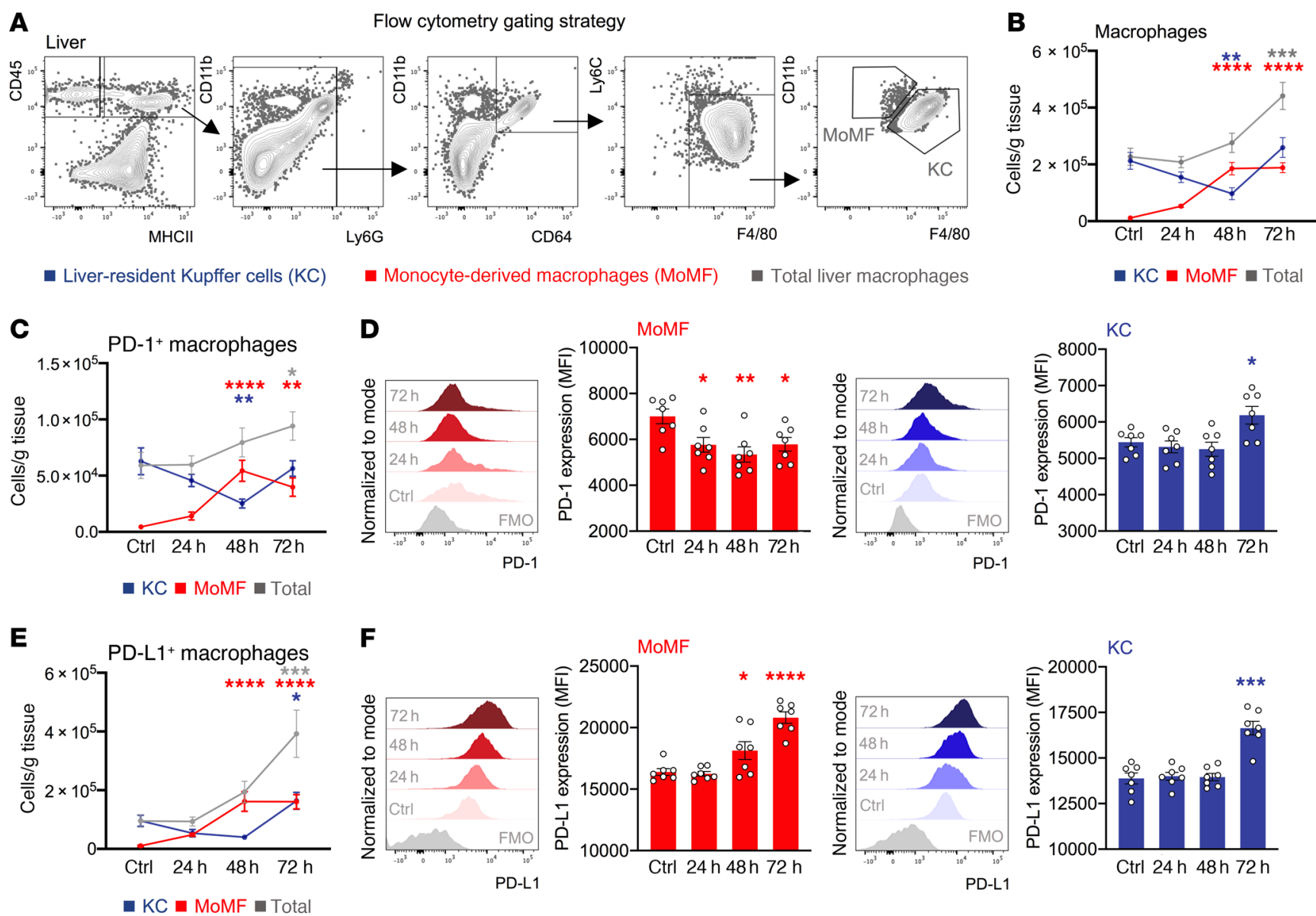

Figure 2. PD-1 and PD-L1 expression in KCs is increased during the resolution of acute liver injury. Hepatic nonparenchymal cells were isolated from livers of baseline (control) and APAP-treated ( 24 h, 48 h, or 72 h) WT mice. Phenotypic characterization of liver CD45+ leukocytes was done by flow cytometry. (A) Representative flow cytometric gating strategy used to identify MoMFs and liver-resident KCs. (B) Number of MoMFs (red), KCs (blue), and total macrophages (gray) ( $n=10-12$ per group). (C) Number of PD-1 MoMFs (red), PD-1+ KCs (blue), and PD-1 $1^{+}$total liver macrophages (gray) ( $n=7$ per group). (D) Representative histograms and data showing PD-1 expression (MFI) by MoMFs and KCs ( $n=7$ per group). (E) Number of PD-L1+ MoMFs (red), PD-L1+ KCs (blue), and PD-L1+ total liver macrophages (gray) ( $n=7$ per group). (F) Representative histograms and data showing PD-L1 expression (MFI) of MoMFs and KCs ( $n=7$ per group). Results are from 3 (B) and 2 (C-F) independent experiments. Each symbol represents an individual mouse. Data are presented as the mean \pm SEM. ${ }^{*} P<0.05,{ }^{* *} P<0.01,{ }^{* * *} P<0.001$, and ${ }^{* * * *} P<0.0001$, by 1 -way ANOVA (compared with control). FMO, fluorescence minus one.

PD-1+ KCs exhibited upregulation of M2-like (Arg1, Cd36, Cd206, Cdh1, Csf1, Fn1) and downregulation of M1-like (Irf5, Il1b, Il18) macrophage polarization genes (Figure 4, D and E, and Supplemental Figure 3). They also had high expression of the suppressors of cytokine signaling Socs 1 and Socs3. Compared with PD-1 $\mathrm{KCs}, \mathrm{PD}-1^{+} \mathrm{KCs}$ were characterized by the downregulation of genes related to lymphocyte regulation (Btk, CD64, Cd80, Cd86, Syk), complement activation, TLR signaling (Itgam, Cd14, Cd80, Cd86, Btk, Irf5, Irf7), and pathogen responses (Aoah, Nlrp3, Il1b, Fpr1, Mpeg1, Ncf2, Cybb) (Figure 4, D and E, and Supplemental Figure 3). To confirm the latter, flow cytometry-sorted PD-1 ${ }^{+}$and PD-1KCs were subjected to an E. coli phagocytosis assay. PD-1 $1^{+}$KCs showed reduced E. coli uptake, providing a functional correlate of the gene expression trends (Figure 4C). These data indicate that PD-1 expression by KCs is associated with an immune-suppressive profile and impaired antimicrobial functions in acute liver injury.

$P D-1^{-/}$mice with acute liver injury show efficient KC bacterial clearance and protection from sepsis. To determine whether the PD-1/PD-L1 axis may alter the inflammatory and antimicrobial responses of KCs after injury, we studied control and APAPtreated WT and PD-1/- mice (Supplemental Figure 4A). Compared with WT mice, $\mathrm{PD}-1^{-/-}$mice had similar ALT levels but reduced hepatic necrosis scores 48 hours and 72 hours after APAP treatment (Supplemental Figure 4, B and C) and showed no differences in blood immune cells or liver monocytes and macrophages (Supplemental Figure 4, D-F, and Supplemental Figure 5, A and B). $\mathrm{PD}-1^{-/}$mice also had more $\mathrm{CD} 8^{+} \mathrm{T}$ cells in the liver, failed to show expanded PD-L1 $1^{\text {hi }}$ NKT cells at resolution, and had reduced liver tissue Pdl1, Cxcl10, and Cxcl16 mRNA levels (Supplemental Figure 5, D and E, and Supplemental Figure $4 \mathrm{H}$ ). Intravital imaging and flow cytometric analysis revealed comparable $\mathrm{KC}$ bacterial capture in control WT and PD-1/- animals 20 minutes after E. coli infection (Figure 5, A-D). In contrast to APAP-treated WT mice, which had reduced KC E. coli capture and killing, APAP-treated PD-1 $1^{-/}$mice showed a preserved KC phagocytic capacity (Figure $5, B-E)$ and a lower tissue bacterial burden, suggesting a lack of systemic bacterial dissemination (20 min after infection, Supplemental Figure 1E). 
A
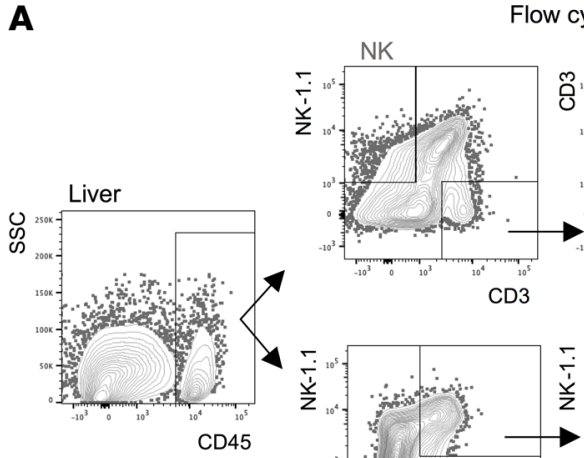

Flow cytometry gating strategy

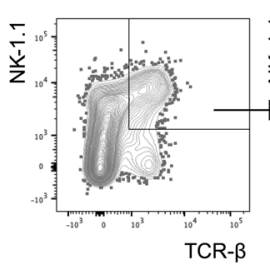

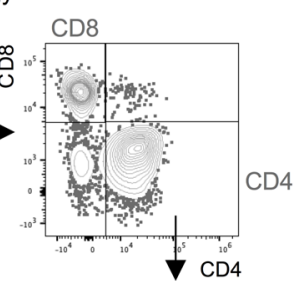

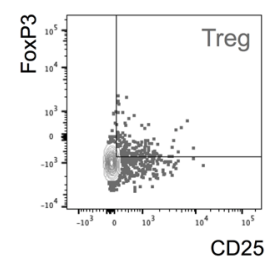

B
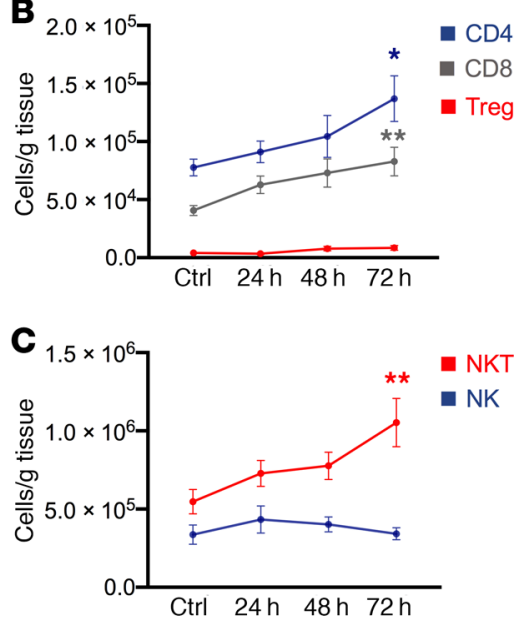
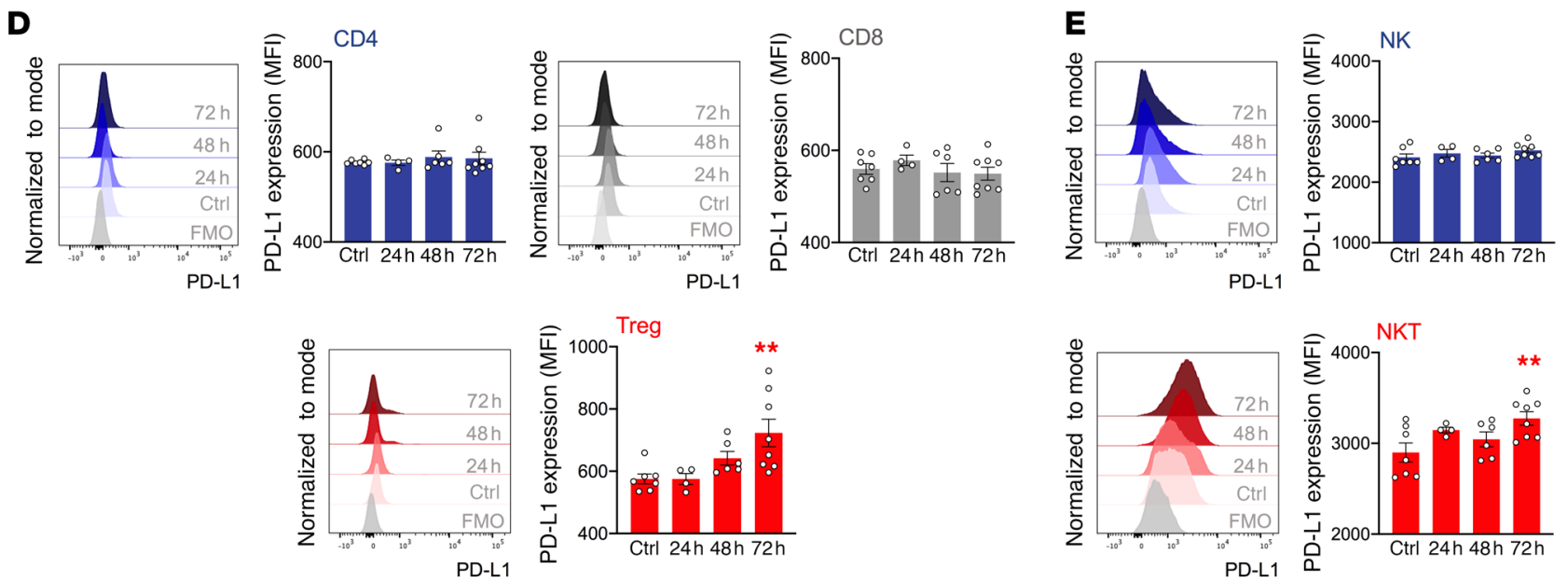

Figure 3. PD-L1 expression of lymphocyte subsets is increased during the resolution of acute liver injury. Hepatic nonparenchymal cells were isolated from livers of baseline (control) and APAP-treated ( 24 h, 48 h, or 72 h) WT mice. Phenotypic characterization of liver CD45+ leukocytes was done by flow cytometry. (A) Representative flow cytometric gating strategy used to identify CD4+ T cells, CD8+ T cells, Tregs, NK cells, and NKT cells. (B) Number of CD4+ T cells (blue), CD8 ${ }^{+}$T cells (gray), and Tregs (red) per gram of tissue ( $n=8-12$ per group). (C) Number of NK (blue) and NKT (red) cells per gram of tissue ( $n$ = 8-12 per group). (D) Representative histograms and data showing PD-L1 expression (MFI) of CD4+ T cells, CD8 ${ }^{+}$T cells, and Tregs ( $n=4-8$ per group). (E) Representative histograms and data showing PD-L1 expression (MFI) of NK and NKT cells ( $n=4-8$ per group). Results are from 3 (B and C) and 2 ( $\mathbf{D}$ and E) independent experiments. Each symbol represents an individual mouse. Data are presented as the mean \pm SEM. ${ }^{*} P<0.05$ and ${ }^{*} P<0.01$, by 1 -way ANOVA (compared with control).

We further sought to evaluate disease severity at later time points after E. coli infection using an established experimental sepsis score (29) and by assessing the tissue bacterial burden and sepsis-related inflammatory markers 24 hours after infection (29-31). Compared with control WT mice, APAP-treated WT mice had significantly higher sepsis scores after infection (Figure $5 \mathrm{~F}$ ) and an increased bacterial load in the liver, spleen, and lungs (Figure 5G). In contrast to APAP-treated WT mice, control and APAP-treated $\mathrm{PD}-1^{-/-}$mice had lower sepsis scores after infection (Figure $5 \mathrm{~F}$ ) and a lower bacterial load in the liver, spleen, and lungs (Figure 5G). In line with these data, APAP-treated WT mice showed an augmented inflammatory response following E. coli infection. Compared with APAP-treated PD-1 ${ }^{-/-}$mice, APAP-treated WT mice had a higher fold change of C-reactive protein (CRP) and lactate levels (Supplemental Figure 6, A and B) and a cytokine profile that favored a proinflammatory imbalance, as indicated by increased sepsis severity markers (29-31) such as IL-6 and a higher IL-6/IL-10 ratio (Supplemental Figure 6, C-F).

PD-1 blockade improves KC bacterial clearance in mice with acute liver injury and confers protection from sepsis. We next questioned whether in vivo blockade of the PD-1/PD-L1 pathway could improve $\mathrm{KC}$ bacterial clearance and prevent sepsis development in mice with liver injury. For this purpose, APAP-treated mice were dosed with a blocking anti-PD-1 Ab or an isotype control 48 hours after APAP treatment (Supplemental Figure 7A). Of note, liver injury indices and proportions of liver immune cell subset were similar between groups (Figure 6, A and B, and Supplemental Figure $7 \mathrm{~B})$. Compared with isotype-treated mice, anti-PD-1-treated mice showed enhanced KC bacterial capture in the liver (Figure 6, C and D, and Supplemental Figure 7, C and D), lower blood E. coli counts (Figure 6E), and reduced tissue bacterial burden (20 min after infection, Supplemental Figure 1E). Later time-point disease monitoring revealed that, compared with isotype-treated mice, 
A

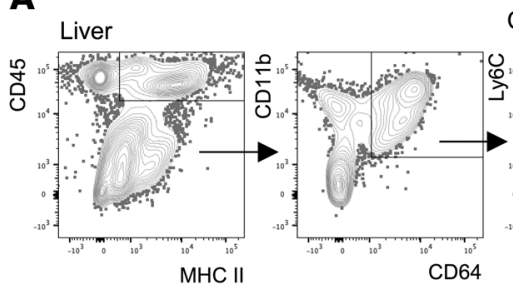

B

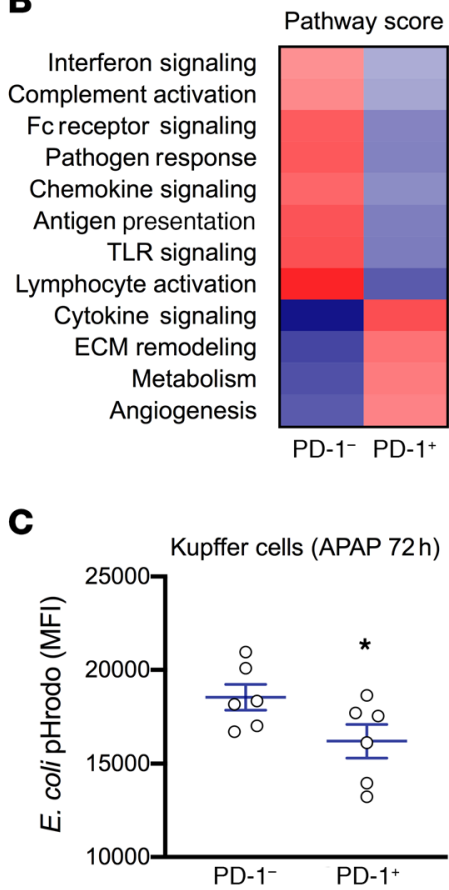

E

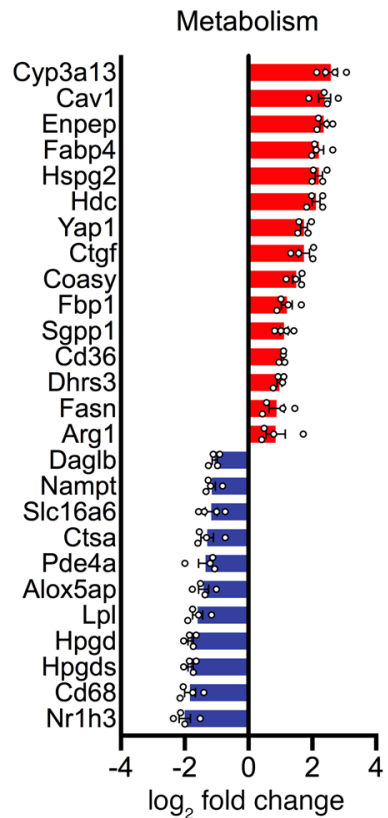

Cell sorting - flow cytometry gating strategy
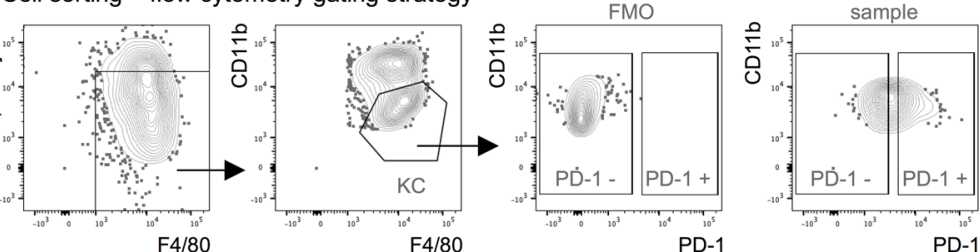

D
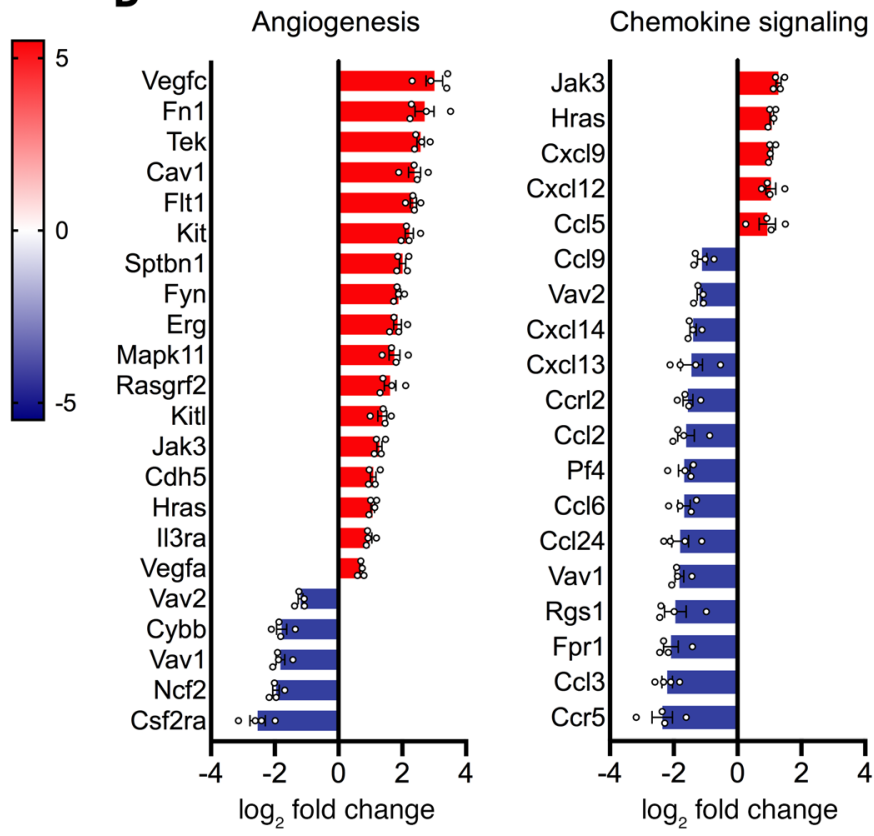

Figure 4. PD-1-expressing KCs exhibit an immunesuppressive profile during the resolution of acute liver injury. $\mathrm{PD}-1^{+}$and $\mathrm{PD}-1^{-} \mathrm{KC}$ subsets were sorted from livers of APAP-treated ( $72 \mathrm{~h}$ ) WT mice using flow cytometry ( $n=4$ each). KC lysates were assessed for mRNA expression using the NanoString nCounter system (Mouse Myeloid Innate Immunity Panel). Data show comparison of $\mathrm{PD}-1^{+}$with $\mathrm{PD}-1^{-}$cells (baseline). (A) Representative flow cytometric gating strategy used to sort the PD-1+ and PD-1- cell subsets. (B) Pathway scoring of PD- $1^{+}$and PD-1- KCs performed using nCounter Advanced Analysis. (C) Phagocytosis (uptake) of E. coli pHrodo was assessed by flow cytometry in $\mathrm{PD}-1^{+}$and PD-1- KC subsets (APAP, $72 \mathrm{~h}$ ) ( $n=6$ per group). ${ }^{*} P<0.05$, by Wilcoxon paired test. Results are from 2 independent experiments. ( $\mathbf{D}$ and $\mathbf{E}$ ) Data show $\log _{2}$ fold change of normalized linear count data (PD- $1^{+}$ subset, $n=4$ ) for significantly differentially expressed genes (based on nCounter Advanced Analysis) in various pathways. A Benjamini-Hochberg $P$ value adjustment was applied. Statistical significance was set at $P<0.05$ and a 2 -fold linear change. Data are presented as the mean \pm SEM. 
A

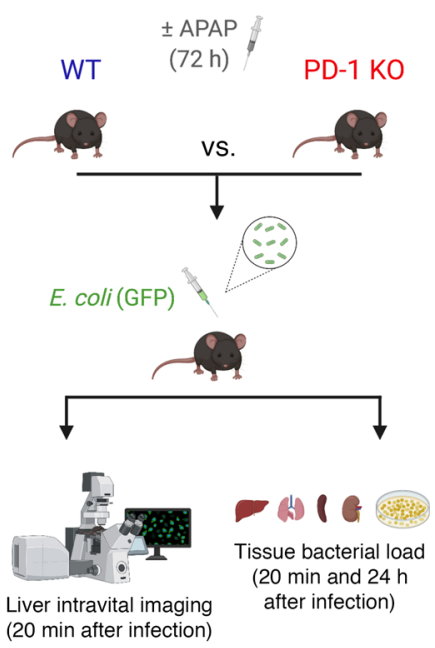

C

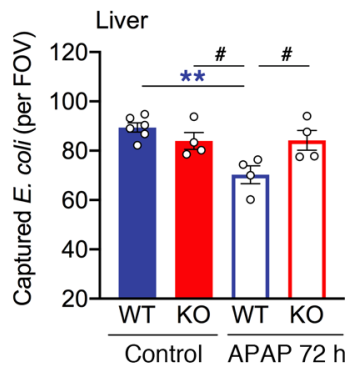

G

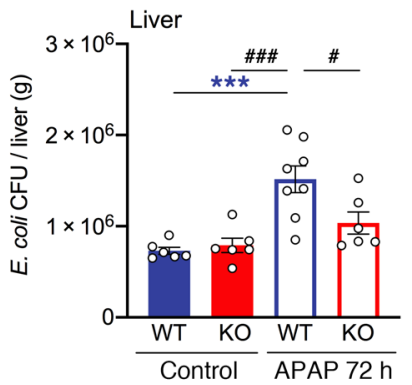

B
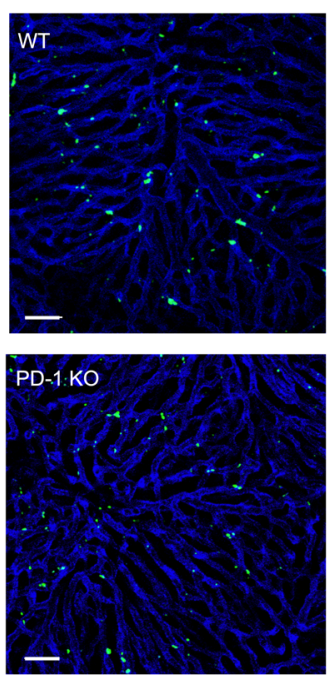

CD31 (endothelium) GFP (E. coli) F4/80 (macrophages)

Control
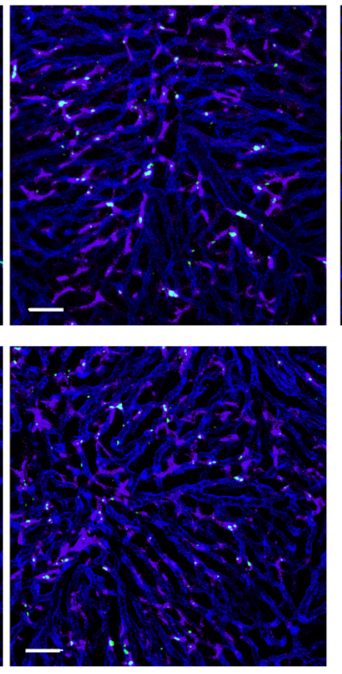

E
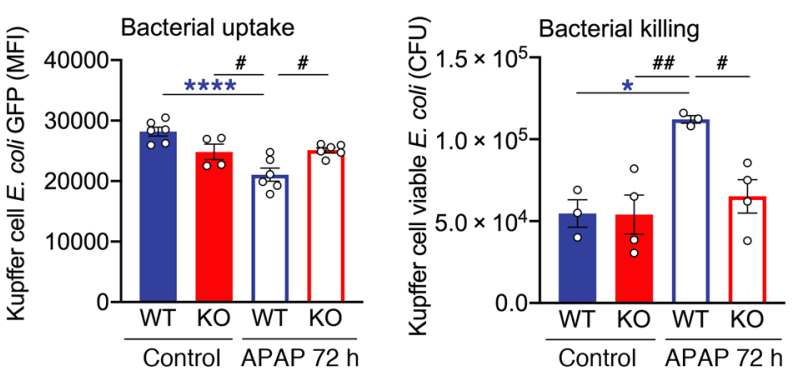

$\mathbf{F}$

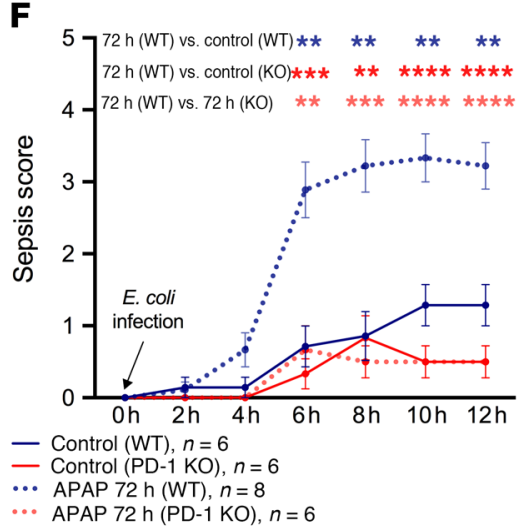

Figure 5. PD-1 deficiency improves KC bacterial clearance and confers protection from sepsis in mice with acute liver injury. Baseline (control) and APAP-treated (72 h) WT and PD-1-deficient (PD-1 KO) mice were intravenously challenged with E. coli. (A) Schematic of experimental E. coli challenge. (B) Representative intravital liver images (20 min after infection). Macrophages and endothelial cells were stained with fluorescently labeled anti-F4/80 (purple) and anti-CD31 (blue) antibodies, respectively; GFP+ E. coli (green). Scale bars: $50 \mu \mathrm{m}$. (C) Intravital liver imaging analysis showing the amount of macrophage-captured E. coli per FOV ( $n=4-6$ per group). (D) KC E. coli uptake measured by flow cytometry ( $n=4-6$ per group). (E) Sorted KCs from control or APAP-treated $(72 \mathrm{~h}) \mathrm{WT}$ and PD-1/- mice were in vitro challenged with E. coli (1:100 ratio, $60 \mathrm{~min})$. In vitro bacterial killing of KC phagocytosed $E$. coli was evaluated by measuring viable $E$. coli CFU recovered from KC lysates ( $n=3-4$ per group). (F) Mouse sepsis scores over time following $E$. coli infection ( $n=$ 6-8 per group). (C) CFU analysis of bacterial burden in the liver, spleen, lungs, and kidneys 24 hours after infection ( $n=6-8$ per group). Results are from 3 $(\mathbf{B}-\mathbf{D}, \mathbf{F}$, and $\mathbf{G})$ and $2(\mathbf{E})$ independent experiments. Each symbol represents an individual mouse. Data are presented as the mean $\pm \mathrm{SEM}$. ${ }^{*}$ or ${ }^{\#} P<0.05,{ }^{* *}$ or ${ }^{\#} P<0.01,{ }^{* *}$ or ${ }^{\# \# \#} P<0.001,{ }^{* * *} P<0.0001$, by 1-way ANOVA (C-E and $\mathbf{C}$ ) or 2-way, repeated-measures ANOVA (F).

anti-PD-1-treated mice had lower sepsis scores after infection (Figure 6F) and a reduced bacterial load in the liver, spleen, and lungs (24 h after infection, Figure 6G), as was seen in $\mathrm{PD}-1^{-/}$mice (Figure 5, F and G). Moreover, anti-PD-1-treated infected mice had unaltered CRP levels, lactate levels, and IL-6/IL-10 ratios compared with control mice (Supplemental Figure 6, C-F). Taken together, these findings demonstrate that PD-1 deficiency or antiPD-1 therapy in mice with liver injury is associated with improved 
A

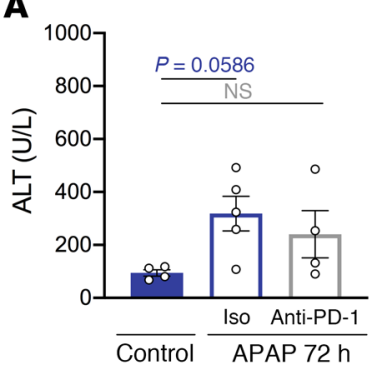

D

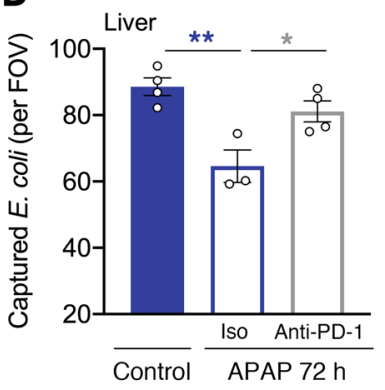

B

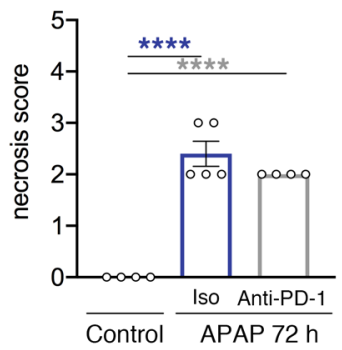

E

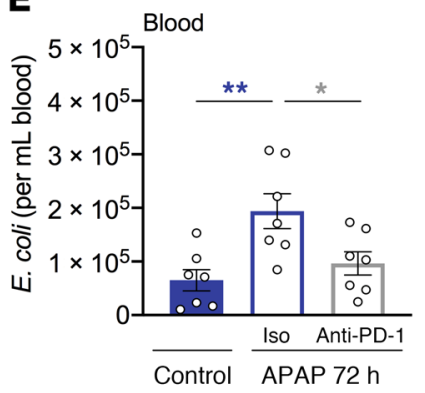

C
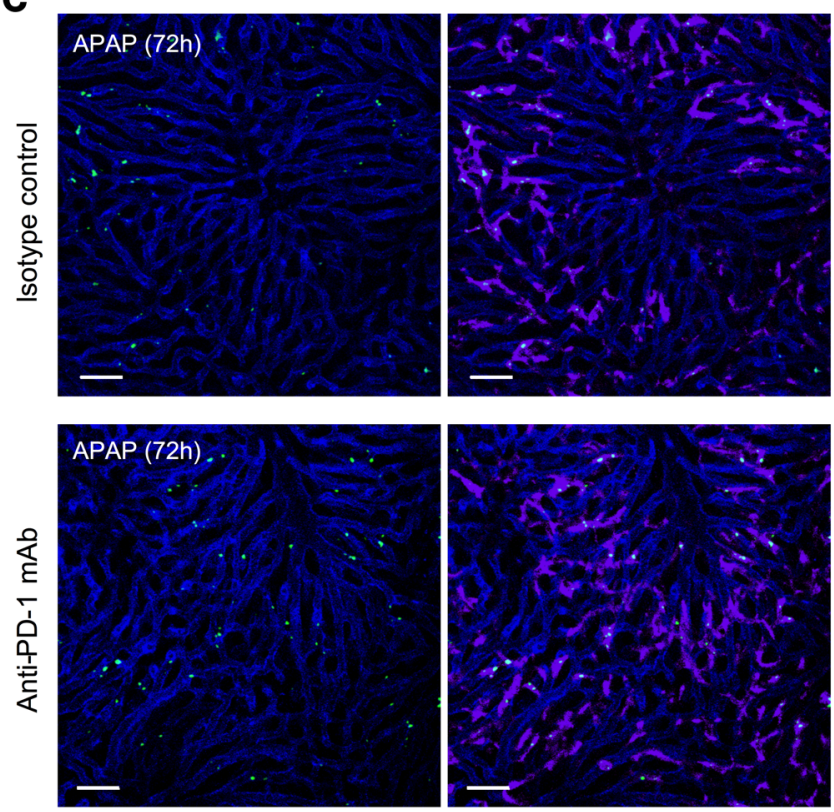

CD31 (endothelium) GFP (escherichia coli) F4/80 (macrophages)
$\mathbf{F}$

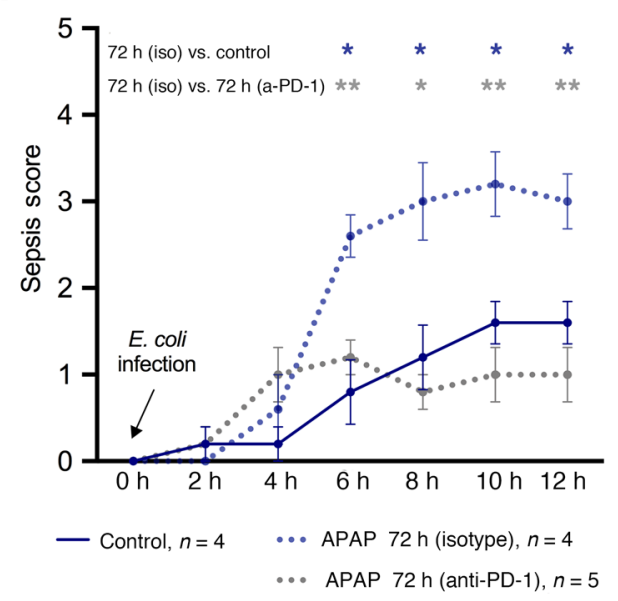

$\mathbf{G}$
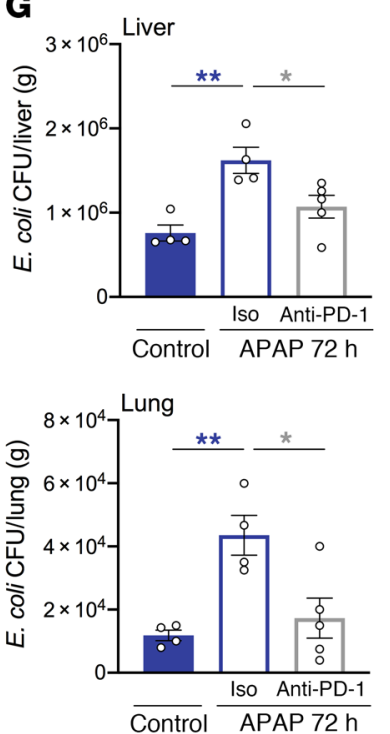
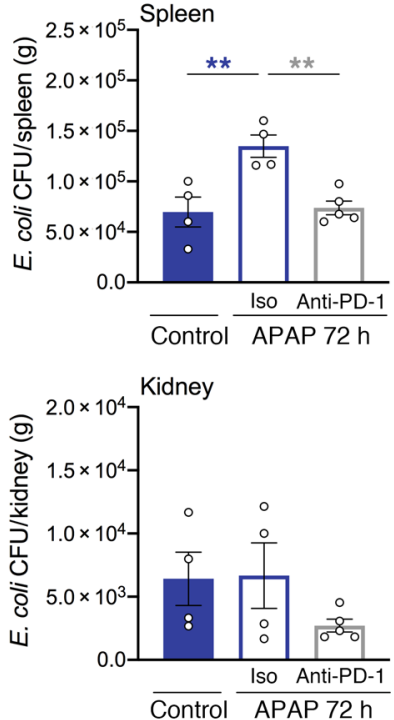

Figure 6. PD-1 blockade improves KC bacterial clearance and confers protection from sepsis in mice with acute liver injury. Baseline (control) and APAP-treated (72 h) WT mice, dosed with isotype control (iso) or anti-PD-1 mAb 48 hours after APAP treatment, were intravenously challenged with E. coli. (A) Plasma alanine transaminase (ALT) levels (U/L) and (B) hepatic necrosis scores ( $n=4-5$ per group). (C) Representative intravital liver images from APAP-treated (72 h) mice (20 min after infection). Macrophages and endothelial cells were stained with fluorescently labeled anti-F4/80 (purple) and anti-CD31 (blue) antibodies, respectively; GFP+ E. coli (green). Scale bars: $50 \mu \mathrm{m}$. (D) Intravital liver imaging analysis showing the amount of macrophage-captured $E$. coli per FOV ( $n=3-4$ per group). (E) Number of free $E$. coli in blood measured by flow cytometry ( $n=7$ per group). (F) Mouse sepsis scores over time following E. coli infection ( $n=4-5$ per group). (C) CFU analysis showing bacterial burden in the liver, spleen, lungs, and kidneys 24 hours after infection ( $n=4-5$ per group). Results in $\mathbf{A}-\mathbf{G}$ are for 2 independent experiments. Each symbol represents an individual mouse. Data are presented as the mean \pm SEM. ${ }^{*} P<0.05,{ }^{* *} P<0.01$, and ${ }^{* * *} P<0.0001$, by 1-way ANOVA (A-E and $\mathbf{G}$ ) or 2-way, repeated-measures ANOVA (F).

KC bacterial clearance, reduced tissue bacterial burden, and protection from sepsis after infection.

Increased lymphocyte PD-L1 expression and soluble PD-L1 plasma levels in patients with ALF. Having established that the PD-1/PD-L1 axis altered KC responses in murine acute liver injury, we sought to demonstrate that this translates to human disease and that the PD-1/PD-L1 pathway may be a potential therapeutic target. We aimed to assess PD-1 and PD-L1 expression in PBMCs from healthy controls (HCs) and from patients with stable CLD or ALF (Table 1). We identified peripheral lymphocyte subsets by flow cytometry (ref. 7, Figure 7A, and Supplemental Figure 8A) and found no differential PD-1 expression by lymphocytes among the study groups (Supplemental Figure 8, A-D). However, PD-L1 expression by $\mathrm{CD}^{+}{ }^{+} \mathrm{T}$ cells, $\mathrm{CD} 8^{+} \mathrm{T}$ cells, and Tregs was signifi- 
A
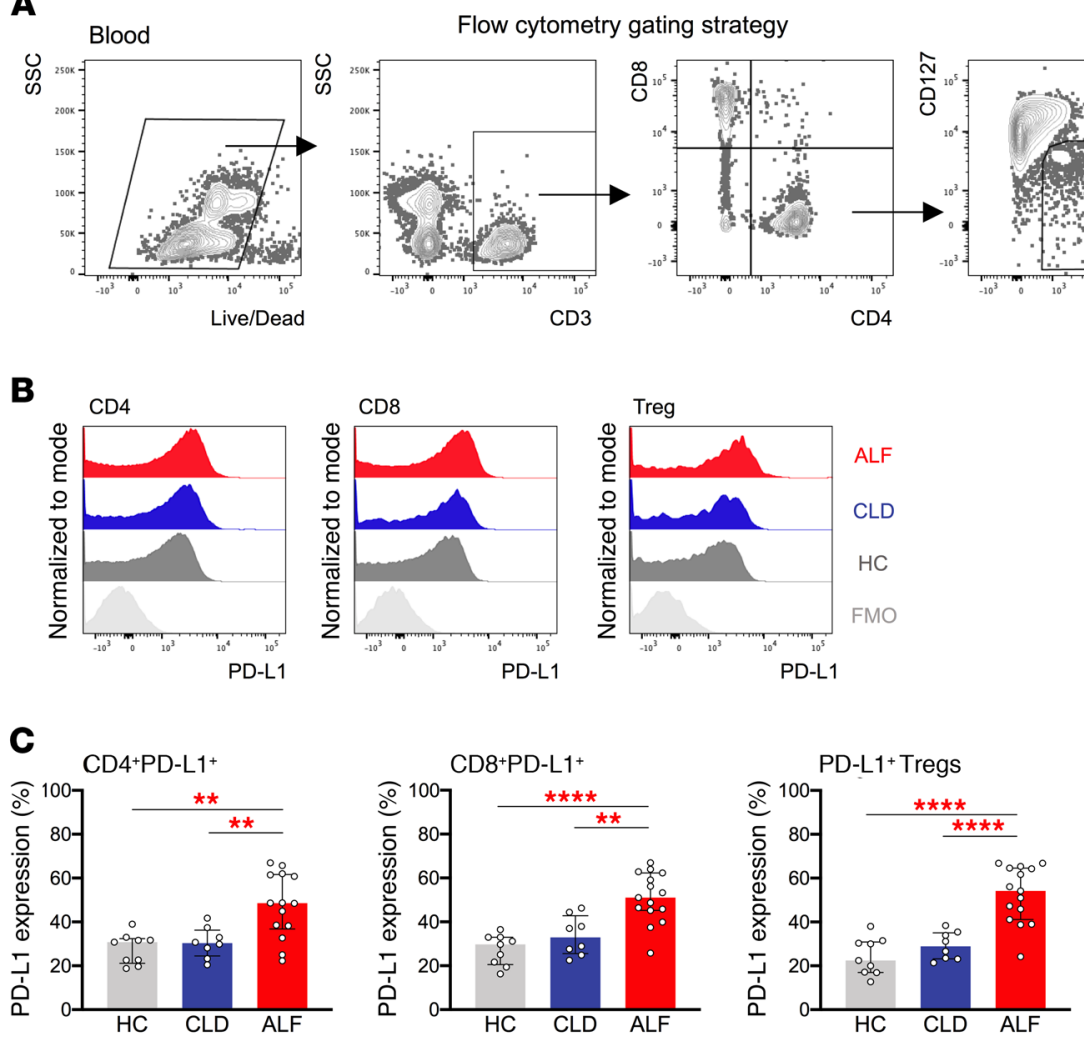
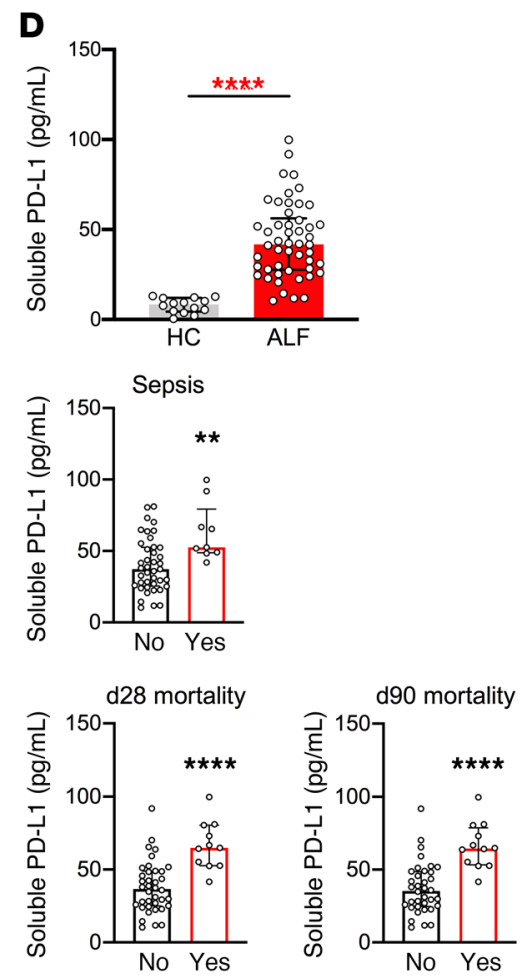

\section{$\mathbf{E}$}
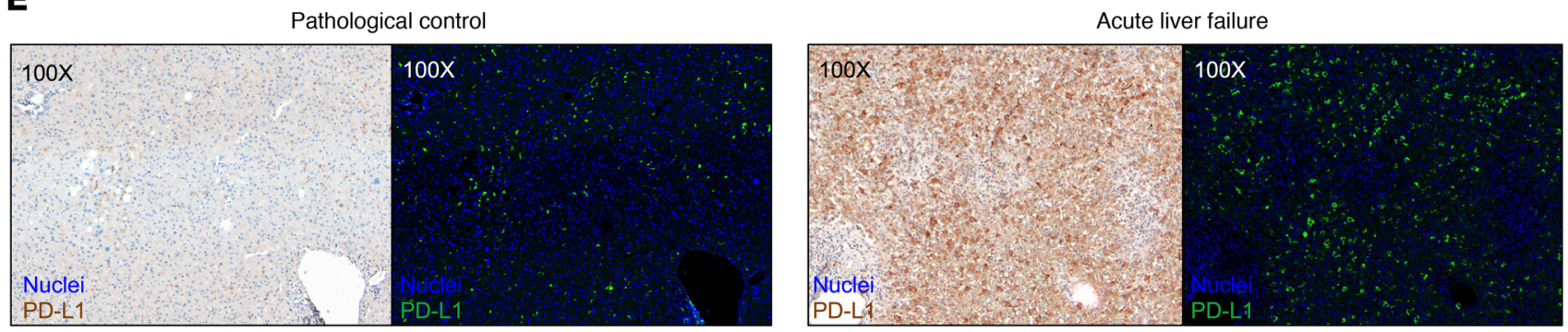

Figure 7. Lymphocyte PD-L1 expression and SPD-L1 plasma levels are increased in patients with ALF. Phenotypic characterization of lymphocytes was performed by flow cytometry in PBMCs from HCs $(n=9)$ and patients with CLD $(n=8)$ or ALF $(n=15)$. (A) Representative flow cytometric gating strategy used to identify CD4 ${ }^{+} \mathrm{T}$ cells, CD8 ${ }^{+} \mathrm{T}$ cells, and Tregs. (B and C) Representative histograms and data showing PD-L1 expression (percentage) in CD4+ ${ }^{+}$cells, CD8 ${ }^{+}$T cells, and Tregs. (D) Dot plots show plasma SPD-L1 levels as determined by ELISA in HCs $(n=8)$ and patients with ALF $(n=50)$ and plasma sPD-L1 levels in patients with ALF based on development of sepsis (no: $n=41$; yes: $n=9$ ), day-28 mortality (no: $n=38$; yes: $n=11$ ), or day-90 mortality (no: $n=36$; yes: $n=12$ ). (E) Representative IHC images of PD-L1 staining in pathological control and APAP-induced ALF liver tissues analyzed using Nuance multispectral imaging technology. Left panels: RGB images show nuclei (blue) and PD-L1 (brown) staining. Original magnification, $\times 100$. Right panels: Pseudofluorescence images show nuclei (blue) and PD-L1 (green) staining. Original magnification, $\times 100$. Data are presented as the median with the IQR. ${ }^{* *} P<0.01$ and ${ }^{* * *} P<0.0001$, by Mann-Whitney $U$ test.

cantly increased in patients with ALF (Figure 7, B and C), in both patients with acetaminophen-induced ALF (AALF) and those with non-acetaminophen-induced ALF (NAALF) (Supplemental Figure 8B). In line with this, IHC showed increased expression of hepatic PD-L1 in ALF explant tissue compared with expression in pathological controls (Figure 7E and Supplemental Figure 8E).

In addition to its major membrane-bound form, a soluble form of PD-L1 (sPD-L1) can be found in plasma and is increased in a few cancers, where it is negatively associated with survival and the response to immunotherapy, suggesting an inhibitory effect $(32,33)$. We detected increased plasma levels of sPD-L1 in patients with ALF compared with levels in HCs (Supplemental Table 1 and Figure 8D). sPD-L1 plasma levels in patients with ALF were positively correlated with the sequential organ failure assessment (SOFA) score, as well as with creatinine and lactate levels, and were negatively correlated with $\mathrm{pH}$ (Supplemental Figure 9D). Notably, plasma sPD-L1 levels were significantly higher in patients with ALF who developed sepsis or had a poor outcome (Figure 7D).

Increased monocyte PD-1 expression in patients with ALF. We identified peripheral monocytes by flow cytometry (ref. 6 and Figure 8A). Compared with HCs and patients with CLD, we 
Table 1. Clinical and physiological parameters of patients with CLD or ALF

\begin{tabular}{|c|c|c|c|}
\hline Parameters & $\mathrm{HCs}$ & CLD & ALF \\
\hline Number & 16 & 8 & 20 \\
\hline Age (yr) & 38 & 56 & $37^{\mathrm{A}}$ \\
\hline $\operatorname{Sex}(M / F)$ & $9 / 7$ & $4 / 4$ & $7 / 13$ \\
\hline Etiology: $n$ & NA & $\begin{array}{l}\text { ALD cirrhosis: } 5 \text {, } \\
\text { NASH cirrhosis: } 2 \text {, } \\
\text { PSC: } 1\end{array}$ & $\begin{array}{c}\text { Drug-induced: } 11 \\
\text { (APAP-induced: } 8 \text {, } \\
\text { mixed overdose: } 3 \text {, } \\
\text { non-drug-induced: } 9\end{array}$ \\
\hline WBC $\left(\times 10^{9} / L\right)$ & NA & $4.25(2.91-7.97)$ & $7.29^{\mathrm{B}}(3.95-15.53)$ \\
\hline Monocytes (× 109/L) & NA & $0.39(0.25-0.62)$ & $0.27(0.09-0.95)$ \\
\hline Neutrophils (× $\left.10^{9} / \mathrm{L}\right)$ & NA & $2.49(0.81-4.85)$ & $6.49^{\circ}(3.36-12.3)$ \\
\hline Lymphocytes (× 109/L) & NA & $1.36(0.53-2.30)$ & $0.90(0.50-2.03)$ \\
\hline Albumin (g/L) & NA & $31.5(27-44)$ & $29.0(19-36)$ \\
\hline AST (IU/mL) & NA & $61(28-92)$ & $1834^{\mathrm{D}}(39-6488)$ \\
\hline Bilirubin ( $\mu \mathrm{mol} / \mathrm{L})$ & NA & $35.0(17-62)$ & $99.5^{\mathrm{B}}(26-522)$ \\
\hline Creatinine $(\mu \mathrm{mol} / \mathrm{L})$ & NA & $77(42-121)$ & $78(26-205)$ \\
\hline INR & NA & $1.46(0.99-1.96)$ & $2.90^{c}(1.00-7.23)$ \\
\hline MELD & NA & $15.1(6-22)$ & $30.2^{\circ}(21-43)$ \\
\hline SOFA & NA & $4(0-5)$ & $5.5^{A}(1-17)$ \\
\hline
\end{tabular}

ALD, alcoholic liver disease; PSC, primary sclerosing cholangitis; NASH, nonalcoholic steatohepatitis; $\mathrm{M}$, male; $\mathrm{F}$, female. Data are presented as median values with the IQR. ${ }^{A} P<0.05$, ${ }^{\mathrm{B}} P<0.01,{ }^{\mathrm{C}} P<0.001$, and ${ }^{\mathrm{D}} P<$ 0.0001 , by Mann-Whitney $U$ test for comparison of ALF with CLD.

found that patients with ALF had increased proportions of PD-1 $1^{+}$ monocytes and PD-1 expression levels (Figure 8B). Of note, the percentage of $\mathrm{PD}-1^{+}$monocytes was higher in patients with AALF than in those with NAALF (Supplemental Figure 9A). PD-1 was detected at high levels in intermediate monocytes (Figure 8C) and was coexpressed with activation/pro-restorative markers in patients with ALF (refs. 4-6 and Supplemental Figure 9C). Also, ALF monocytes had higher PD-L1 expression (Supplemental Figure 9B), mirroring our murine macrophage data (Figure 2, E and F). We consistently observed increased expression of hepatic PD-1 and PD-L1 in ALF explant tissue, with more pronounced expression in AALF tissue, compared with pathological controls (Figure 7E, Supplemental Figure 8E, and Supplemental Figure 10, A and B). Monocyte PD-1 expression correlated positively with SOFA scores and lactate levels and negatively with $\mathrm{pH}$ and peripheral monocyte numbers (Figure 8D). Interestingly, PD-1 expression was higher in patients with ALF who developed sepsis or had a poor outcome (Supplemental Figure 9E).

$P D-1$ in vitro blockade improves monocyte bacterial uptake in human ALF. Compared with HCs and patients with CLD, monocytes from patients with ALF showed impaired E. coli phagocytosis (ref. 8 and Supplemental Figure 9F). We found that E. coli uptake was lower in $\mathrm{PD}-1^{+}$versus $\mathrm{PD}-1^{-}$monocytes in patients with ALF and that monocyte PD-1 expression inversely correlated with the phagocytosis index (Supplemental Figure 9F). We therefore explored the effects of anti-PD-1 treatment on PBMCs from HCs and patients with ALF. We demonstrate that PD- 1 in vitro blockade restored monocyte $E$. coli uptake in patients with ALF (Figure 8E) and increased LPS-stimulated proinflammatory cytokines (Supplemental Figure 9G). Together, our data suggest that monocyte PD-1 expression and plasma sPD-L1 levels may be used as prognostic biomarkers and that PD-1 blockade can restore monocyte innate responses in ALF.

\section{Discussion}

Our study reports impaired KC bacterial clearance and systemic bacterial dissemination after $E$. coli infection in mice with acute liver injury. We describe a role of the PD-1/PD-L1 pathway in regulating $\mathrm{KC}$ inflammatory and antimicrobial responses. We demonstrate that the tolerogenic effects of this axis involve PD- $1^{+}$ KCs and $\mathrm{PD}-\mathrm{L} 1^{+}$lymphocyte subsets that may provide suppressive back signaling. Gene expression profiling of $\mathrm{PD}-1^{+} \mathrm{KCs}$ revealed an immune-suppressive profile typified by reduced antigen presentation, lymphocyte activation, and pathogen responses. Notably, PD-1 deficiency or PD-1 blockade in mice with liver injury improved $\mathrm{KC}$ bacterial clearance, reduced the tissue bacterial burden, and conferred protection from sepsis after infection.

The regulatory role of $\mathrm{PD}-1 / \mathrm{PD}-\mathrm{L} 1$ signaling in $\mathrm{T}$ cell function and differentiation is well documented $(18,19)$, however, accumulating evidence has associated PD-1 expression by macrophages with immune suppression in infection, sepsis, and cancer (23-27). Here, in the context of sterile liver inflammation, we found that PD- 1 expression by KCs was enhanced during the resolution phase. PD-1 expression can be induced on monocytes and macrophages by TLR ligands (e.g., LPS) and various cytokines (TNF- $\alpha$, IL-1 $\beta$, and IL-6; refs. 21, 22). Such inflammatory cues are highly present in the hepatic microenvironment after injury $(3,17,34)$ and could therefore be responsible for PD-1 induction in KCs.

Recent studies showed that PD-1+ ${ }^{+}$TAMs exhibit an M2-like expression $\left(\mathrm{CD} 206^{\mathrm{hi}} \mathrm{MHC}\right.$ class $\mathrm{II}^{\mathrm{lo}} \mathrm{CD} 64^{\mathrm{lo}}$ ) profile and phagocytose fewer tumor cells or OVA in colon and gastric cancer, respectively, compared with PD-1 ${ }^{\text {neg }}$ TAMs $(26,35)$. Here, we provide an in-depth transcriptomic profile of $\mathrm{PD}-1^{+} \mathrm{KCs}$ during the resolution of liver injury. Consistent with the above data $(26,35)$, we found that PD-1+ KCs exhibited M2-like polarization (e.g., Arg1, Cd206) and immune-suppressive properties characterized by a reduction in antigen presentation and lymphocyte costimulation-related molecules (e.g., Btk, Cd64, Cd80, Cd86). Furthermore, PD- $1^{+} \mathrm{KCs}$ showed reduced expression of TLR signaling factors (e.g., Irf5, Irf7), antimicrobial defense molecules, including enzymes (e.g., Aoah, Mpeg1), and inflammasome-related factors (e.g., Nlrp3, $I l 1 b, I l 18)$. In addition, $\mathrm{PD}-1^{+} \mathrm{KCs}$ have lower expression of $C y b b$ and $N c f 2$ genes that encode the membrane protein gp91phox and cytosolic subunit p67-phox of the NADPH oxidase complex, respectively. This complex is crucial for the production of ROS in phagocytes, and its reduction implies an inability of $\mathrm{PD}-1^{+}$cells to kill the ingested pathogens. This is consistent with our data showing impaired KC bacterial killing during the resolution of injury. Altogether, our study demonstrates that PD-1 expression by $\mathrm{KCs}$ is associated with an immune-suppressive profile and reduced antimicrobial functions.

PD-1/PD-L1 signaling is crucial in regulating $\mathrm{T}$ cell responses $(18,19)$. We found that hepatic PD-L1 ${ }^{+}$lymphocyte subsets were expanded during the resolution of liver injury in mice. Likewise, in humans, we detected increased expression of peripheral lympho- 
A
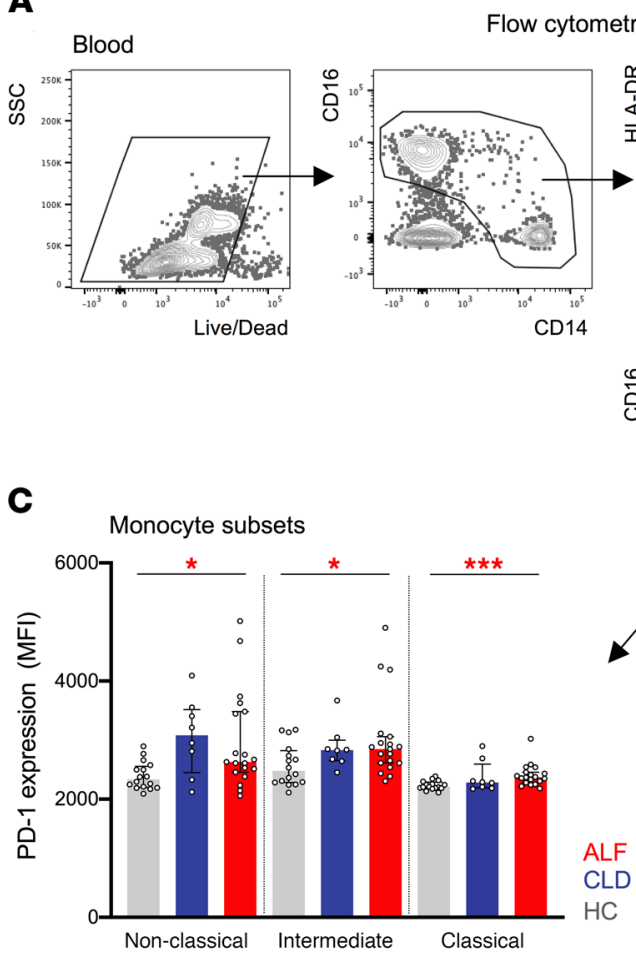

E
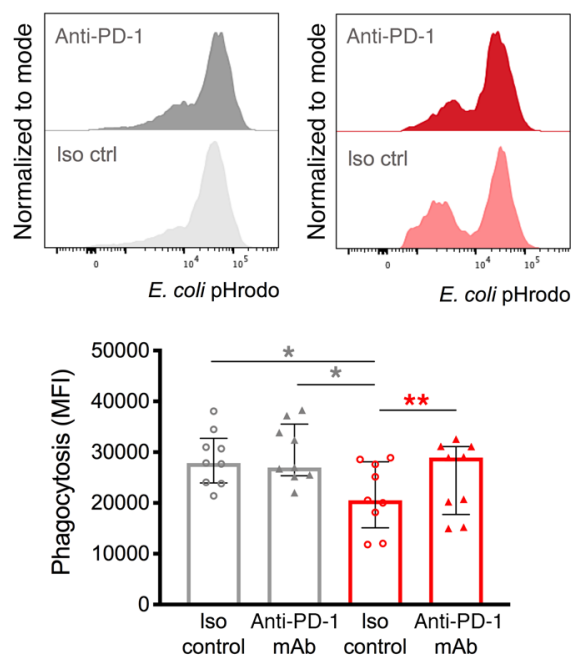

Flow cytometry gating strategy
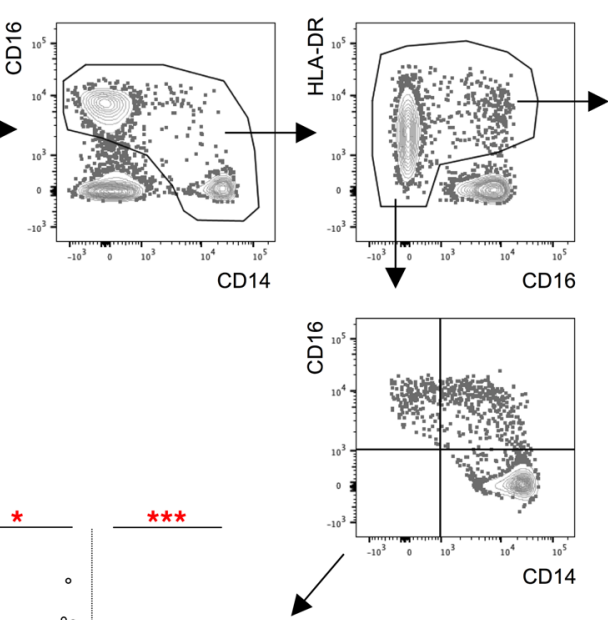

B
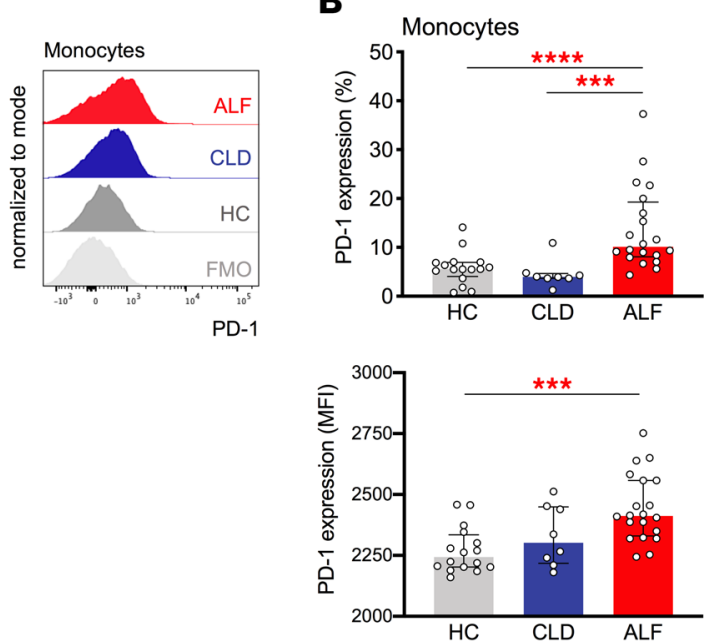

D

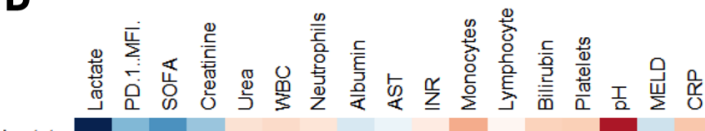

Lactate
PD.1..MFI.
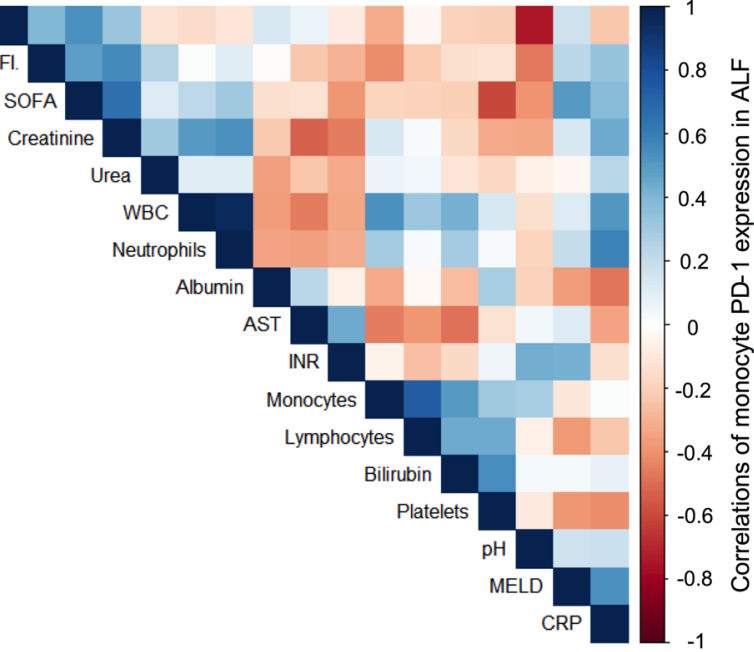

\section{峞}

Creatinine

Figure 8. Monocyte PD-1 expression is increased in patients with ALF. Phenotypic characterization of monocytes was performed by flow cytometry in PBMCs from HCs $(n=16)$ and patients with CLD $(n=8)$ or ALF $(n=20)$. (A) Representative flow cytometric gating strategy used to identify monocytes and determine their PD-1 expression. Histograms show PD-1 expression in HCs and in patients with CLD or ALF. (B and C) PD-1 expression levels in (B) total monocytes and (C) monocyte subsets (classical, intermediate, and nonclassical). Statistical significance was determined by Mann-Whitney $U$ test. (D) Matrix of correlation of monocyte PD-1 expression (MFI) with clinical scores and biochemical parameters for patients with ALF. INR, international normalized ratio; AST, aspartate aminotransferase; MELD, model for end-stage liver disease; WBC, white blood cell count. (E) PBMCs from HCs and patients with ALF were cultured in the presence of $10 \%$ autologous plasma and treated with anti-PD-1 mAb $(10 \mu \mathrm{g} / \mathrm{mL})$ or an isotype-matched control (iso ctrl) ( $10 \mu \mathrm{g} /$ $\mathrm{mL}$ ) prior to E. coli pHrodo phagocytosis assay. Flow cytometric plots and analysis show $E$. coli pHrodo phagocytosis levels (MFI) in HCs and patients with $\operatorname{ALF}\left(n=9\right.$ per group). Results are from 3 independent experiments. Data are presented as the median with the IQR. ${ }^{*} P<0.05,{ }^{* *} P<0.01,{ }^{* * *} P<0.001$, and ${ }^{* * *} P<0.0001$, by Wilcoxon paired test (ALF group) and Mann-Whitney $U$ test (HCs versus patients with ALF).

cyte PD-L1, hepatic PD-L1, and plasma SPD-L1 in patients with ALF. Reports have shown that PD-L1 in T cells can be induced in response to antigen presentation and sterile inflammatory cues (e.g., IFN- $\gamma$, IL-4), whereas PD-L1 ${ }^{+} \mathrm{T}$ cells can lead to tumorpromoting tolerance $(28,36)$. PD-L1+ $\mathrm{T}$ cells suppress neighbor- ing effector T cells via the PD-L1/PD-1 axis and promote STAT6dependent, M2-like tolerogenic macrophages in cancer (28). These findings are functionally relevant to our model, as the increase in PD-L1 ${ }^{+}$lymphocytes and SPD-L1 after injury may exert suppressive effects on $\mathrm{PD}-1^{+}$KCs. Further work, for example in 
coculture experiments, could examine the effects of lymphocytes on monocyte and $\mathrm{KC}$ function.

KCs are important for maintaining liver tolerance. During homeostasis, $\mathrm{KC}$-associated antigen presentation induces $\mathrm{CD} 4^{+}$ $\mathrm{T}$ cell liver arrest and expansion of $\mathrm{IL}^{-}-0^{+}$Tregs, however, this immunological tolerance is abrogated in chronic liver injury and fibrosis (37). Of note, in vivo interactions of liver-patrolling NKT cells with $\mathrm{KCs}$ are observed during steady-state and after infection (38-40). Here, we found PD-L1 ${ }^{\text {hi }}$ NKT cells and Tregs during resolution $(39,41)$ of sterile acute liver injury. We speculate that these PD-L1 ${ }^{\text {hi }}$ subsets interfacing with $\mathrm{PD}-1^{+} \mathrm{KC}$ may exert suppressive effects on the latter via the PD-1/PD-L1 axis. Future studies will need to delineate the in vivo interactions of lymphocytes with $\mathrm{KCs}$ during homeostasis and liver inflammation and further examine the PD-1/PD-L1 pathway and its regulatory role in the liver.

We found impaired $\mathrm{KC}$ bacterial clearance after liver injury and reduced pathogen responses of $\mathrm{PD}-1^{+} \mathrm{KCs}$, in line with other studies that have associated PD-1 with macrophage suppression. For instance, $\mathrm{PD}-1^{-/-}$mice are protected from septic peritonitis, displaying improved bacterial clearance and a less severe cytokine storm (23), whereas PD-1 or PD-L1 mAb blockade leads to similar results $(42,43)$. We consistently found that both PD-1 ${ }^{-/-}$mice and anti-PD-1 mAb-treated mice with liver injury had unimpaired $\mathrm{KC}$ bacterial clearance and were protected from sepsis after systemic infection. Importantly, in mouse models, PD-1 deficiency or PD-1 mAb blockade did not exaggerate liver injury or delay its resolution, suggesting that PD-1 therapy may be a safe strategy to prevent immune suppression after liver injury. Strauss et al. found that PD-1 in myeloid cells dampened antitumor immunity and demonstrated that myeloid cell PD-1 (PD-1 fl/flLysMcre $\left.^{\mathrm{f}}\right)$ ablation was as effective as global PD-1 $\left(\mathrm{PD}-1^{-/}\right)$ablation in limiting tumor growth, and considerably more effective than lymphocyte PD-1 (PD-1 $\left.1^{\mathrm{f} / \mathrm{flCD} 4 \mathrm{cre}}\right)$ ablation (27). Given this recent evidence, the lack of such experimental models should be acknowledged as a limitation in our study. Future research using mice with myeloid- or KC-specific PD-1 ablation should examine in more detail the role of PD- 1 in regulating the function of liver macrophages.

Peripheral monocyte dysfunction and its contribution to infection susceptibility is documented in patients with ALF. For instance, monocytes exhibit a reduction in proinflammatory cytokines and bacterial uptake $(4-6,8)$. PD-1 expression by monocytes is reportedly increased in human chronic lymphocytic leukemia (25), HIV or active tuberculosis $(22,24)$, and sepsis (23), whereas PD-1/PD-L1 blockade restores innate responses in sepsis $(23,44,45)$. In conjunction with our murine liver findings, we report that the PD-1/PD-L1 pathway may contribute to peripheral monocyte suppression in human ALF, as we detected increased systemic levels of monocyte PD-1, lymphocyte PD-L1, and sPD-L1. We consistently observed increased hepatic PD-1 and PD-L1 expression in ALF. Of note, monocyte PD-1 and sPD-L1 levels were markedly higher at hospital admission in patients who had sepsis and a poor outcome and therefore may serve as prognostic biomarkers. Future prospective studies, including longitudinal patient sampling, are warranted to assess the utility of these markers in predicting immune suppression and mortality in patients with liver injury.
Immune checkpoint inhibition has recently been considered a therapeutic strategy in CLD. Defects in adaptive and humoral immunity can be partially rescued in vitro by PD-1 blockade, as shown for T cells in alcohol-related liver disease (46) and for B cells in viral hepatitis B infection (47). The safety and efficacy of PD-1/ PD-L1 mAb blockade for the treatment of hepatocellular carcinoma has also been assessed in clinical trials, with promising results (48-50). However, immune checkpoint inhibition in myeloid cells and its contribution to the efficiency of such mAb-based therapies remain unexplored. Furthermore, patients with liver cirrhosis have a high risk of infection and mortality (51). Bacterial infections occur frequently in patients with cirrhosis (32\%-34\%, refs. 51, 52) and are the main precipitant for hepatic acute decompensation $(\mathrm{AD})$, with or without organ failure (acute-on-chronic liver failure $[\mathrm{ACLF}])(53,54)$. Like ALF $(3)$, cirrhosis and ACLF are associated with inflammation and, as disease progresses, immune dysfunction that contribute to increased susceptibility to infection $(5,8,55-57)$. It would be important to further investigate the significance of the PD-1/PD-L1 pathway in cirrhosis, AD, and ACLF and its contribution to systemic and hepatic immune suppression.

In conclusion, we describe a crucial role of the PD-1/PD-L1 axis in regulating $\mathrm{KC}$ and monocyte inflammatory and antimicrobial responses following acute liver injury. Our findings suggest that PD-1-targeted immunotherapy may be a strategy to prevent innate immune suppression in ALF.

\section{Methods}

Mice. C57BL/6 WT mice were purchased from Charles River Labora-

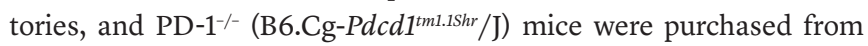
The Jackson laboratory. Mice were housed and bred at the Imperial College London animal facility under specific pathogen-free conditions. For induction of sterile acute liver injury, male mice (aged 8-12 weeks) were fasted overnight and received an intraperitoneal injection of $250 \mathrm{mg} / \mathrm{kg}$ APAP (MilliporeSigma) diluted in saline. WT mice received an intraperitoneal injection of either $200 \mu \mathrm{g}$ anti-PD-1 mAb (clone RMP1-14, BioXCell) or $200 \mu \mathrm{g}$ IgG2a control (clone 2A3, BioXCell) diluted in saline.

Patients. Patients with ALF $(n=50)$ (Table 1 and Supplemental Table 1) were recruited to the study within 24 hours of their admission to the Liver Intensive Therapy Unit (LITU) of King's College Hospital. Inpatients with CLD $(n=8)$ and HC volunteers $(n=16)$ (Table 1) were recruited and served as pathological controls and HCs, respectively. Liver tissue was obtained from patients with ALF $(n=4)$ undergoing orthotopic liver transplantation (OLT), and pathological control tissue was derived from hepatic resection margins of colorectal malignancies $(n=3)$. Exclusion criteria included age younger than 18 years, neoplasia, and immunosuppressive therapy. Biochemical parameters were determined on a hematological analyzer (Advia 2120, Siemens).

Intravital microscopy. Intravital imaging of mice was performed by confocal microscopy. For imaging of bacterial capture, hepatic macrophages and endothelial cells were visualized by intravenous injection of anti-F4/80-BV421 (5 $\mu \mathrm{g} /$ mouse, BM8, BioLegend) and anti-CD31-phycoerythrin (anti-CD31-PE) (5 $\mu \mathrm{g} / \mathrm{mouse,} \mathrm{MEC13.3,}$ BioLegend) antibodies. Mice were anesthetized by intraperitoneal injection of ketamine/medetomidine $(125 / 1 \mathrm{mg} / \mathrm{kg})$. After a midabdominal incision was made, the mouse was placed in a right lateral 
position, and the largest liver lobe was externalized on a coverslip attached to the microscope stage. The liver was covered with saline-moistened lab wipes to restrict movement, and the microscope heating chamber was kept at $37^{\circ} \mathrm{C}$ to maintain mouse body temperature. The liver was then imaged with an inverted Leica TCS SP5 confocal microscope with the Leica DM6000 Confocal Fixed Stage system. Bacteria that remained associated with KCs for more than 2 minutes were considered captured. Time-lapse images were recorded for up to 20 minutes after infection. A total of 5-6 field of view (FOV) images were recorded for each mouse under a $\times 20$ microscope objective, and their average was considered $1 \mathrm{FOV}$. Videos and images were processed and analyzed with Imaris 8 software (Bitplane).

Bacterial challenge. E. coli GFP (25922GFP, American Type Culture Collection [ATCC]) bacteria were cultivated in Tryptic Soy Agar/Broth (MilliporeSigma). For flow cytometry, mice received an intravenous dose of $5 \times 10^{6} \mathrm{E}$. coli GFP (per $20 \mathrm{~g}$ BW, ref. 10). Five minutes after E. coli injection, $10 \mu \mathrm{L}$ blood was obtained, diluted (1:40) in PBS, and analyzed on a BD LSRFortessa Cell Analyzer (BD Biosciences). The number of $\mathrm{GFP}^{+}$events was used to calculate free E. coli in the circulation using 123count eBeads Counting Beads (Invitrogen, Thermo Fisher Scientific). For intravital imaging of E. coli capture in the liver, mice received an intravenous dose of $5 \times 10^{7}$ E. coli GFP (per $20 \mathrm{~g}$ BW, ref. 10) and were imaged under confocal microscopy for $20 \mathrm{~min}-$ utes after infection. For sepsis studies, mice received an intravenous nonlethal dose of $5 \times 10^{7}$ E. coli GFP bacteria (per $20 \mathrm{~g} \mathrm{BW}$ ). A murine sepsis scoring system (29) was used to evaluate disease severity. Animal health monitoring was conducted by 2 investigators every 2 hours after infection (for 12 hours), with evaluation of appearance, level of consciousness, activity, response to stimuli, eyes, and respiration rate and quality (29). For evaluation of tissue bacterial burden, organs were harvested, weighed, and homogenized in $1 \mathrm{~mL}$ PBS. Tissue suspensions were plated onto agar plates at serial dilutions, and CFU were counted after an 18 -hour incubation at $37^{\circ} \mathrm{C}$.

Bacterial killing assay. Bacterial killing of $\mathrm{KCs}$ was measured in cell lysate fractions (58). Briefly, sorted KCs (20,000 cells) from control and APAP-treated $(72 \mathrm{~h})$ mice were incubated in 48-well, flatbottomed plates $\left(37^{\circ} \mathrm{C}, 5 \% \mathrm{CO}_{2}\right)$ with $E$. coli GFP (ATCC $25922 \mathrm{GFP}$ ) at a 1:100 ratio in DMEM (Gibco, Thermo Fisher Scientific) supplemented with $10 \%$ autologous plasma. After a 60-minute incubation, the plates were put on ice, and supernatants were aspirated. Cell pellets were next lysed in distilled water ( $\mathrm{pH} 11)$, lysate fractions were plated onto agar plates at serial dilutions, and CFU were counted after an 18 -hour incubation at $37^{\circ} \mathrm{C}$.

Flow cytometry. Mouse hepatic nonparenchymal cells were isolated (14) for phenotypic characterization of myeloid and lymphoid cell subsets by flow cytometry. Human PBMCs were isolated as previously described (6). Blood monocytes and lymphoid cell subsets were phenotypically characterized by flow cytometry. Details on the Abs used for flow cytometric staining are provided in the supplemental material. Data acquisition was performed on a BD LSRFortessa Cell Analyzer (BD Biosciences). Flow cytometric data analysis was performed using FlowJo, version 10.

NanoString nCounter. KCs from APAP-treated WT mice (72 h) were FACS-sorted using a FACSAria Fusion Analyzer (BD Biosciences). mRNA expression was analyzed with the NanoString nCounter Mouse Myeloid Innate Immunity Panel (734 immunology-related and 20 housekeeping genes). Data were analyzed using nCounter Advanced Analysis software (NanoString Technologies). Normalization (geNorm) and $P$ value adjustment (Benjamini-Hochberg) were applied, as detailed in the Supplemental Methods. Statistically significant results were considered at a $P$ value of less than 0.05 and a fold change of 2 .

For further technical information, see the supplemental materials.

Statistics. Data graph presentation and statistical analyses were performed using GraphPad Prism 8 (GraphPad Software). Data are presented as the mean \pm SEM, unless stated otherwise. A MannWhitney $U$ nonparametric test was used for statistical analysis of differences between 2 groups. A 1-way ANOVA with Dunnett's or Tukey's multiple-comparison test was used for analysis of differences between more than 2 groups. A 2-way ANOVA with Tukey's multiple-comparison test was used for analysis of differences between more than 2 groups and with 2 different independent variables. A Spearman's nonparametric correlation test was used for associations with clinical parameters. A $P$ value of less than 0.05 was considered statistically significant.

Study approval. All animal experimental protocols were approved by the Imperial College London in accordance with UK Home Office regulations (PPL: P8999BD42). The human study was approved by the UK National Health Service (NHS) Health Research Authority (LREC: 12/LO/0167, 15/LO/0363). Assent was given by the patients' nominated next of kin if the patients were unable to provide informed consent themselves.

\section{Author contributions}

ET, CGA, and MRT conceptualized the study. ET, CLCG, and MAM performed mouse experiments. ET, CLCG, MAM, HCH, FMT, MKS, TO, HK, CB, SKM, RDG, and MJWM collected, analyzed, and interpreted data. ET, WK, LAP, KJW, and MRT wrote the original draft of the manuscript. ET, CLCG, MKS, MB, WK, MJWM, LAP, KJW, JAW, RDG, and MRT wrote and critically revised the manuscript. ET, CGA, and MRT acquired funding.

\section{Acknowledgments}

We express our gratitude to Dr. Charalambos (Harry) Antoniades for his important contribution to this project. We will always remember him and carry on his enthusiasm for research.

We also gratefully acknowledge all the patients and healthy volunteers who participated in this study. We thank the LMS/ National Institute for Health Research (NIHR) Flow Facility at the Imperial College London for cell-sorting services and the University College London NanoString Facility for providing the nCounter system and related services. The graphical abstract and figure graphs were created with BioRender (https://biorender.com/). This project was supported by the Rosetrees Trust (M439-F1, to ET, CGA, and MRT), the Imperial College London (Imperial College Research Fellowship Award, to ET), and the UK NIHR and the NIHR Imperial Biomedical Research Centre (BRC). MRT is the recipient of an NIHR Senior Investigator award (NIHR200153).

Address correspondence to: Evangelos Triantafyllou, Imperial College London, Section of Hepatology and Gastroenterology, St. Mary's Hospital, W2 1NY, London, UK. Email: e.triantafyllou@ imperial.ac.uk. 
1. Stravitz RT, Lee WM. Acute liver failure. Lancet. 2019;394(10201):869-881.

2. Bernal W, et al. Acute liver failure: A curable disease by 2024? J Hepatol. 2015;62(S1):S112-S120.

3. Triantafyllou E, et al. The role of monocytes and macrophages in acute and acute-on-chronic liver failure. Front Immunol. 2018;9:2948.

4. Antoniades CG, et al. Secretory leukocyte protease inhibitor: a pivotal mediator of anti-inflammatory responses in acetaminophen-induced acute liver failure. Hepatology. 2014;59(4):1564-1576.

5. Bernsmeier C, et al. Patients with acute-onchronic liver failure have increased numbers of regulatory immune cells expressing the receptor tyrosine kinase MERTK. Gastroenterology. 2015;148(3):603-615.e14.

6. Triantafyllou E, et al. MerTK expressing hepatic macrophages promote the resolution of inflammation in acute liver failure. Gut. 2018;67(2):333-347.

7. Khamri W, et al. Increased expression of cytotoxic T-lymphocyte-associated protein 4 by $\mathrm{T}$ cells, induced by $\mathrm{B} 7$ in sera, reduces adaptive immunity in patients with acute liver failure. Gastroenterology. 2017;153(1):263-276.e8.

8. Bernsmeier C, et al. CD $14^{+} \mathrm{CD}^{-} 5^{-} \mathrm{HLA}^{-D R}$ myeloid-derived suppressor cells impair antimicrobial responses in patients with acute-onchronic liver failure. Gut. 2018;67(6):1155-1167.

9. Krenkel O, Tacke F. Liver macrophages in tissue homeostasis and disease. Nat Rev Immunol. 2017;17(5):306-321.

10. Nakagaki BN, et al. Immune and metabolic shifts during neonatal development reprogram liver identity and function. J Hepatol. 2018;69(6):1294-1307.

11. Zeng Z, et al. CRIg functions as a macrophage pattern recognition receptor to directly bind and capture blood-borne gram-positive bacteria. Cell Host Microbe. 2016;20(1):99-106.

12. Zeng Z, et al. Sex-hormone-driven innate antibodies protect females and infants against EPEC infection. Nat Immunol. 2018;19(10):1100-1111.

13. Balmer ML, et al. The liver may act as a firewall mediating mutualism between the host and its gut commensal microbiota. Sci Transl Med. 2014;6(237):237ra66-237ra66.

14. Graubardt N, et al. Ly6C ${ }^{\text {hi }}$ monocytes and their macrophage descendants regulate neutrophil function and clearance in acetaminopheninduced liver injury. Front Immunol. 2017;8:626.

15. Zigmond $\mathrm{E}$, et al. Infiltrating monocyte-derived macrophages and resident kupffer cells display different ontogeny and functions in acute liver injury. J Immunol. 2014;193(1):344-353.

16. Mossanen JC, et al. Chemokine (C-C motif) receptor 2-positive monocytes aggravate the early phase of acetaminophen-induced acute liver injury. Hepatology. 2016;64(5):1667-1682.

17. Antoniades CG, et al. Source and characterization of hepatic macrophages in acetaminophen-induced acute liver failure in humans. Hepatology. 2012;56(2):735-746.

18. Sharpe AH, Pauken KE. The diverse functions of the PD1 inhibitory pathway. Nat Rev Immunol. 2018;18(3):153-167.

19. LaFleur MW, et al. Inhibitors of the PD-1 pathway in tumor therapy. JImmunol. 2018;200(2):375-383.

20. Qin W, et al. The diverse function of PD-1/
PD-L pathway beyond cancer. Front Immunol. 2019;10:2298.

21. Bally APR, et al. NF- $\kappa$ B regulates PD-1 expression in macrophages. JImmunol. 2015;194(9):4545-4554.

22. Said EA, et al. Programmed death-1-induced interleukin-10 production by monocytes impairs $\mathrm{CD}^{+} \mathrm{T}$ cell activation during HIV infection. Nat Med. 2010;16(4):452-459.

23. Huang $X$, et al. PD-1 expression by macrophages plays a pathologic role in altering microbial clearance and the innate inflammatory response to sepsis. Proc Natl Acad Sci U S A. 2009;106(15):6303-6308.

24. Shen L, et al. PD-1/PD-L pathway inhibits M.tb-specific CD4 ${ }^{+}$T-cell functions and phagocytosis of macrophages in active tuberculosis. $\mathrm{Sci}$ Rep. 2016;6:38362.

25. Qorraj M, et al. The PD-1/PD-L1 axis contributes to immune metabolic dysfunctions of monocytes in chronic lymphocytic leukemia. Leukemia. 2017;31(2):470-478.

26. Gordon SR, et al. PD-1 expression by tumourassociated macrophages inhibits phagocytosis and tumour immunity. Nature. 2017;545(7655):495-499.

27. Strauss L, et al. Targeted deletion of PD-1 in myeloid cells induces antitumor immunity. Sci Immunol. 2020;5(43):1-15.

28. Diskin B, et al. PD-L1 engagement on T cells promotes self-tolerance and suppression of neighboring macrophages and effector T cells in cancer. Nat Immunol. 2020;21(4):442-454.

29. Shrum B, et al. A robust scoring system to evaluate sepsis severity in an animal model. BMC Res Notes. 2014;7(1):233.

30. Ruiz S, et al. Sepsis modeling in mice: ligation length is a major severity factor in cecal ligation and puncture. Intensive Care Med Exp. 2016;4(1):22.

31. Seymour CW, et al. Murine sepsis phenotypes and differential treatment effects in a randomized trial of prompt antibiotics and fluids. Crit Care. 2019;23(1):384.

32. Daassi D, et al. The importance of exosomal PDL1 in tumour immune evasion. Nat Rev Immu nol. 2020;20(4):209-215.

33. Zhu X, Lang J. Soluble PD-1 and PD-L1: predictive and prognostic significance in cancer. Oncotarget. 2017;8(57):97671-97682.

34. Antoniades CG, et al. The importance of immune dysfunction in determining outcome in acute liver failure. J Hepatol. 2008;49(5):845-861.

35. Wang F, et al. Tumor-derived exosomes induce $\mathrm{PD}^{+}$macrophage population in human gastric cancer that promotes disease progression. Oncogenesis. 2018;7(5):41.

36. Wu Y, et al. PD-L1 distribution and perspective for cancer immunotherapy-blockade, knockdown, or inhibition. Front Immunol. 2019;10:2022.

37. Heymann F, et al. Liver inflammation abrogates immunological tolerance induced by Kupffer cells. Hepatology. 2015;62(1):279-291.

38. Geissmann F, et al. Intravascular immune surveillance by CXCR6+ NKT cells patrolling liver sinusoids. PLoS Biol. 2005;3(4):0650-0661.

39. Liew PX, et al. iNKT cells orchestrate a switch from inflammation to resolution of sterile liver injury. Immunity. 2017;47(4):752-765.e5.

40. Lee WY, et al. An intravascular immune response to Borrelia burgdorferi involves Kupffer cells and iNKT cells. Nat Immunol. 2010;11(4):295-302.

41. Martin-Murphy BV, et al. Increased susceptibility of natural killer T-cell-deficient mice to acetaminophen-induced liver injury. Hepatology. 2013;57(4):1575-1584.

42. Brahmamdam $P$, et al. Delayed administration of anti-PD-1 antibody reverses immune dysfunction and improves survival during sepsis. J Leukoc Biol. 2010;88(2):233-240.

43. Zhang Y, et al. PD-L1 blockade improves survival in experimental sepsis by inhibiting lymphocyte apoptosis and reversing monocyte dysfunction. Crit Care. 2010;14(6):1-9.

44. Patera AC, et al. Frontline Science: Defects in immune function in patients with sepsis are associated with PD-1 or PD-L1 expression and can be restored by antibodies targeting PD-1 or PD-L1. J Leukoc Biol. 2016;100(6):1239-1254.

45. Shankar-Hari M, et al. Early PREdiction of sepsis using leukocyte surface biomarkers: the ExPRES-sepsis cohort study. Intensive Care Med 2018;44(11):1836-1848.

46. Markwick LJL, et al. Blockade of PD1 and TIM3 restores innate and adaptive immunity in patients with acute alcoholic hepatitis. Gastroenterology. 2015;148(3):590-602.e10.

47. Salimzadeh L, et al. PD-1 blockade partially recovers dysfunctional virus-specific $B$ cells in chronic hepatitis B infection. JClin Invest. 2018;128(10):4573-4587.

48. El-Khoueiry AB, et al. Nivolumab in patients with advanced hepatocellular carcinoma (CheckMate 040): an open-label, non-comparative, phase $1 / 2$ dose escalation and expansion trial. Lancet. 2017;389(10088):2492-2502.

49. Zhu AX, et al. Pembrolizumab in patients with advanced hepatocellular carcinoma previously treated with sorafenib (KEYNOTE-224): a non-randomised, open-label phase 2 trial. Lancet Oncol. 2018;19(7):940-952.

50. Finn RS, et al. Pembrolizumab as second-line therapy in patients with advanced hepatocellular carcinoma in KEYNOTE-240: a randomized, double-blind, phase III trial. J Clin Oncol. 2020;38(3):193-202.

51. Arvaniti V, et al. Infections in patients with cirrhosis increase mortality four-fold and should be used in determining prognosis. Gastroenterology. 2010;139(4):1246-1256.

52. Gustot T, et al. Impact of infection on the prognosis of critically ill cirrhotic patients: results from a large worldwide study. Liver Int. 2014;34(10):1496-1503.

53. Moreau R, et al. Acute-on-chronic liver failure is a distinct syndrome that develops in patients with acute decompensation of cirrhosis. Gastroenterology. 2013;144(7):1426-1437.

54. Fernández J, et al. Bacterial and fungal infections in acute-on-chronic liver failure: prevalence, characteristics and impact on prognosis. Gut. 2017;67(10):1870-1880.

55. Albillos A, et al. Cirrhosis-associated immune dysfunction: distinctive features and clinical relevance. J Hepatol. 2014;61(6):1385-1396. 
56. Lebossé $\mathrm{F}$, et al. $\mathrm{CD} 8^{+} \mathrm{T}$ cells from patients with cirrhosis display a phenotype that may contribute to cirrhosis-associated immune dysfunction. EBioMedicine. 2019;49:258-268.
57. Brenig R, et al. Expression of AXL receptor tyrosine kinase relates to monocyte dysfunction and severity of cirrhosis. Life Sci Alliance. 2020;3(1):e201900465.
58. Vergis N, et al. Defective monocyte oxidative burst predicts infection in alcoholic hepatitis and is associated with reduced expression of NADPH oxidase. Gut. 2017;66(3):519-529. 